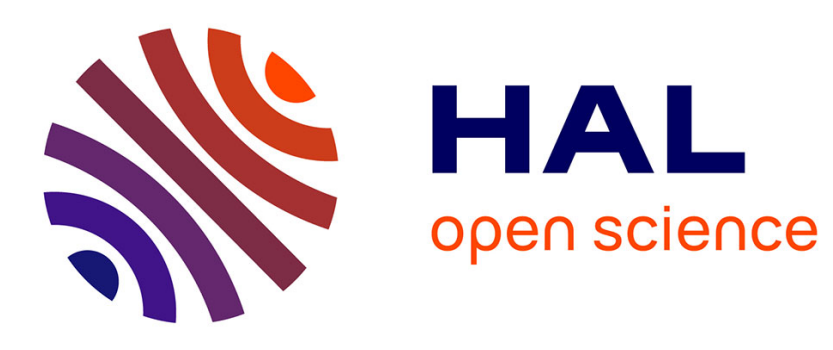

\title{
Interactions between moderately close circular inclusions: the Dirichlet-Laplace equation in the plane
}

Virginie Bonnaillie-Noël, Marc Dambrine

\section{To cite this version:}

Virginie Bonnaillie-Noël, Marc Dambrine. Interactions between moderately close circular inclusions: the Dirichlet-Laplace equation in the plane. Asymptotic Analysis, 2013, 84 (3-4), pp.197-227. 10.3233/ASY-131174 . hal-00680404

\section{HAL Id: hal-00680404 https://hal.science/hal-00680404}

Submitted on 19 Mar 2012

HAL is a multi-disciplinary open access archive for the deposit and dissemination of scientific research documents, whether they are published or not. The documents may come from teaching and research institutions in France or abroad, or from public or private research centers.
L'archive ouverte pluridisciplinaire HAL, est destinée au dépôt et à la diffusion de documents scientifiques de niveau recherche, publiés ou non, émanant des établissements d'enseignement et de recherche français ou étrangers, des laboratoires publics ou privés. 


\title{
Interactions between moderately close circular inclusions: the Dirichlet-Laplace equation in the plane
}

\author{
V. Bonnaillie-Noël* and M. Dambrine ${ }^{\dagger}$
}

March 19, 2012

\section{Introduction}

The presence of small inclusions or of a surface defect modifies the solution of the Laplace equation posed in a reference domain $\Omega_{0}$. If the characteristic size of the perturbation is small, then one can expect that the solution of the problem posed on the perturbed geometry is close to the solution of the reference shape. Asymptotic expansion with respect to that small parameter -the characteristic size of the perturbation- can then be performed.

The case of a single inclusion $\omega$, centered at the origin 0 being either in $\Omega_{0}$ or in $\partial \Omega_{0}$, has been deeply studied, see $[15,16,10,11,18,5,6]$. The techniques rely on the notion of profile, a normalized solution of the Laplace equation in the exterior domain obtained by blow-up of the perturbation. It is used in a fast variable to describe the local behavior of the solution in the perturbed domain. In usually dealt situations that is for Neumann boundary conditions in dimension greater than two and Dirichlet boundary condition in dimension greater than three, convergence of the asymptotic expansion is obtained thanks to the decay of the profile at infinity.

The case of several inclusions was considered for example in the series of papers of A. Movchan and V. Maz'ya [13, 14] where an asymptotic approximation of Green's function is built and justified in a domain with many inclusions. The points where the inclusion is dug are fixed in those works. In [4], the Neumann case where the distance between the holes tends to zero but remains large with respect to their characteristic size was investigated for two perfectly insulated inclusions: a complete multiscale asymptotic expansion of the solution to the Laplace equation is obtained in a three scales case.

We consider in the present work the case of two defects with Dirichlet boundary conditions in a bidimensional domain. For the simplicity of the presentation, we assume that the defects we are considering are disks. Let $\Omega_{0}$ be a bounded domain of $\mathbb{R}^{2}$ containing the origin 0 . For $\varepsilon>0$, small enough, we define the perturbed domain $\Omega_{\varepsilon}$ as

$$
\Omega_{\varepsilon}=\Omega_{0} \backslash \overline{\left(\omega_{\varepsilon}^{-} \cup \omega_{\varepsilon}^{+}\right)}, \text {with } \omega_{\varepsilon}^{ \pm}=x_{\varepsilon}^{ \pm}+\varepsilon \mathcal{B}(0,1),
$$

where $x_{\varepsilon}^{ \pm}= \pm \eta_{\varepsilon} \mathbf{d}$ with a given unitary vector $\mathbf{d}$, and a real number $\eta_{\varepsilon}$. Shortly, $\Omega_{\varepsilon}$ consists of $\Omega_{0}$ from which two $\varepsilon$-disks at distance $2 \eta_{\varepsilon}$ have been removed. We aim at building an

\footnotetext{
*IRMAR, ENS Cachan Bretagne, Univ. Rennes 1, CNRS, UEB, av. Robert Schuman, F-35170 Bruz, France

${ }^{\dagger}$ LMAP, Université de Pau et des Pays de l'Adour, CNRS, F-64013 Pau, France
} 
asymptotic expansion of the solution $u_{\varepsilon}$ of the Laplace problem in $\Omega_{\varepsilon}$ :

$$
\left\{\begin{array}{rll}
-\Delta u_{\varepsilon}=f & \text { in } \Omega_{\varepsilon}, \\
u_{\varepsilon}=0 & \text { on } \Gamma=\partial \Omega_{0}, \\
\partial_{\mathbf{n}} u_{\varepsilon}=0 & \text { on } \partial \omega_{\varepsilon}^{ \pm},
\end{array}\right.
$$

for some $\mathrm{L}^{2}$ datum $f$ whose support does not contain the origin 0 .

We will consider two unstudied cases: $\eta_{\varepsilon}$ is constant and $\eta_{\varepsilon}=\varepsilon^{\alpha}$ for $\alpha \in(0,1)$ :

- In the first case, we are considering two small holes of size $\varepsilon$ around two fixed points $A$ and $B$ and the distance between both is hence fixed. For the cases of Neumann boundary condition or of Dirichlet boundary conditions in dimension at least three, this cases can be treated by separating each hole through cut-off functions and hence reducing it to the single inclusion case. Here, the presence of the logarithmic term prohibits this approach and the interaction between the holes has to be studied.

- In the second case, the distance between $A$ and $B$ collapses to 0 with $\varepsilon$ like $\varepsilon^{\alpha}$, that is to say slower than the size $\varepsilon$ of the inclusions. The interaction between the two holes are then stronger and we will prove that the leading order of the asymptotic expansion is then modified.

This work is hence organized as follows. In a first section, we consider the well studied case of a single inclusion. We explain why the usual method fails in this case, we reformulate ideas of presented in $[15,16,13]$ in order to explain both the strategy we shall follow in the cases of two inclusions and the difficulties we will have to face. In a second step, we consider the case of two defects around two fixed points. We then consider the case of weakly interacting defects: that is to say the distance between the centers of the defects is not fixed but varies like $\varepsilon^{\alpha}$ with the small parameter $\varepsilon$, we show that the structure of the expansion is modified by this interaction.

\section{Single inclusion case}

\subsection{Default of the usual method}

We consider a smooth bounded domain $\Omega_{0}$ such that the origin is contained in $\Omega$. Let $f \in \mathrm{L}^{2}\left(\Omega_{0}\right)$ be such that $0 \notin \operatorname{supp} f$. For $\varepsilon$ small enough such that $\varepsilon \mathcal{B}(0,1) \cap \operatorname{supp} f=\varnothing$, we define a perturbed domain

$$
\Omega_{\varepsilon}=\Omega_{0} \mid \omega_{\varepsilon} \quad \text { with } \quad \omega_{\varepsilon}:=\varepsilon \mathcal{B}(0,1) .
$$

We are interested in describing $u_{\varepsilon}$ the solution of

$$
\left\{\begin{aligned}
-\Delta u_{\varepsilon}= & f \text { in } \Omega_{\varepsilon}, \\
u_{\varepsilon}= & 0 \text { on } \partial \Omega_{\varepsilon} .
\end{aligned}\right.
$$

Since the $\mathrm{H}^{1}$-capacity of $\omega_{\varepsilon}$ tends to 0 as $\varepsilon$ tends to 0 (like $1 / \ln \varepsilon$ ), the function $u_{\varepsilon}$ tends to $u_{0}$ defined as the solution in the unperturbed domain:

$$
\left\{\begin{array}{rll}
-\Delta u_{0}=f & \text { in } \Omega_{0}, \\
u_{0}=0 & \text { on } \partial \Omega_{0} .
\end{array}\right.
$$


To catch the asymptotic of $u_{\varepsilon}$, let us study the error $r_{\varepsilon}^{0}:=u_{\varepsilon}-u_{0}$. It solves

$$
\left\{\begin{aligned}
-\Delta r_{\varepsilon}^{0} & =0 & & \text { in } \Omega_{\varepsilon}, \\
r_{\varepsilon}^{0} & =0 & & \text { on } \partial \Omega_{0}, \\
r_{\varepsilon}^{0} & =-u_{0} & & \text { on } \partial \omega_{\varepsilon} .
\end{aligned}\right.
$$

The solution $u_{0}$ of the unperturbed problem (2.2) is analytic inside $\Omega_{0}$. Hence, it can be approximated in the vicinity of the origin by its Taylor expansion:

$$
u_{0}(x)=u_{0}(\varepsilon X)=u_{0}(0)+\sum_{k \geq 1} D^{k} u_{0}(0)[X, \ldots, X] \frac{\varepsilon^{k}}{k !}, \quad \forall x=\varepsilon X \in \partial \omega_{\varepsilon} .
$$

Each term of this expansion can be seen as an error of order $k$ : the usual idea to improve the expansion of $u_{\varepsilon}$ is now to lift in an harmonic way each term. In the 3-dimension case or for Neumann boundary conditions, this asymptotics analysis is well studied (see for example $[15,16,4] \ldots)$. The key point is the notion of profile, a normalized solution of the Laplace equation in the exterior domain obtained by blow-up of the perturbation. It turns out that profiles tend to 0 at infinity at least in the above mentioned cases. In the converse, for the case considered here (Dirichlet condition in dimension 2), the crucial property is not satisfied: it supposes that the boundary value problem

$$
\left\{\begin{aligned}
-\Delta V & =0 & & \text { in } \mathbb{R}^{2} \backslash \bar{\omega} \\
V & =u_{0}(0) & & \text { on } \partial \omega \\
V & \rightarrow 0 & & \text { at infinity }
\end{aligned}\right.
$$

has a solution. Here $\omega$ is a smooth bounded domain of $\mathbb{R}^{2}$. Unfortunately, this is not the case except when $u_{0}(0)=0$. The classical analysis of elliptic equation in unbounded domain is made in the functional setting of weighted Sobolev spaces, see [2]. It is known that (2.3) has a unique solution in a space containing the constants, hence this solution is the constant $u_{0}(0)$ which prohibits the condition at infinity if $u_{0}(0) \neq 0$.

The existence, uniqueness and behavior at infinity of the solution for a general boundary condition in $\mathrm{H}^{1 / 2}(\partial \omega)$ is given by the following Lemma.

Lemma 2.1 1. Let $\omega$ be a smooth bounded domain of $\mathbb{R}^{2}$ with $0 \in \omega$. Let $f \in \mathrm{H}^{1 / 2}(\partial \omega)$. Then the boundary value problem

$$
\left\{\begin{array}{rll}
-\Delta V & =0 & \text { in } \mathbb{R}^{2} \backslash \bar{\omega} \\
V & =f & \text { on } \partial \omega,
\end{array}\right.
$$

admits a unique weak solution $V$ in the variational space

$$
\mathrm{W}_{0}^{1,2}\left(\mathbb{R}^{2} \backslash \bar{\omega}\right)=\left\{u ; \frac{u}{(1+|X|) \ln (2+|X|)} \in \mathrm{L}^{2}\left(\mathbb{R}^{2} \backslash \bar{\omega}\right) \text { and } \nabla u \in \mathrm{L}^{2}\left(\mathbb{R}^{2} \backslash \bar{\omega}\right)\right\}
$$

2. Furthermore, the solution $V$ can be decomposed

$$
V(X)=V_{0}+\sum_{k=1}^{n} V_{k}(X)+\mathcal{O}_{\infty}\left(|X|^{-n+1}\right)
$$

where $V_{0}$ is a constant and $V_{k}(X)=\mathcal{V}_{k}(\theta)|X|^{-k}$ where $\theta$ is the usual polar angle and $\mathcal{V}_{k} \in \operatorname{Span}(\cos k \theta, \sin k \theta)$. 
3. If $\omega$ is the unit ball $\mathcal{B}(0,1)$, and if $\int_{\partial \mathcal{B}(0,1)} f=0$, then the solution $V$ reads

$$
V(X)=\sum_{k=1}^{n} V_{k}(X)+\mathcal{O}_{\infty}\left(|X|^{-n+1}\right) \quad \text { with } \quad V_{k}(r, \theta)=\frac{a_{k}(f) \cos k \theta+b_{k}(f) \sin k \theta}{r^{k}} .
$$

In particular, there exists a constant $C$ such that

$$
|V(X)| \leq \frac{C}{|X|} \quad \text { and } \quad|\nabla V(X)| \leq \frac{C}{|X|^{2}} .
$$

\section{Proof:}

1. This result is proved by Giroire in [9].

2. The solution $V$ has a trace on the circle $\partial \mathcal{B}(0, R)$ such that $\omega \subset \mathcal{B}(0, R)$. Let us decompose this trace in Fourier series:

$$
v(\theta)=a_{0}(v)+\sum_{k \geq 1}\left(a_{k}(v) \cos k \theta+b_{k}(v) \sin k \theta\right) .
$$

The restriction of $V$ to the complement $\mathbb{R}^{2} \backslash \mathcal{B}(0, R)$ solves

$$
\left\{\begin{array}{rll}
-\Delta V & =0 & \text { in } \mathbb{R}^{2} \backslash \overline{\mathcal{B}(0, R)}, \\
V & =v & \text { on } \partial \mathcal{B}(0, R)
\end{array}\right.
$$

and hence reads

$$
V(r, \theta)=a_{0}(v)+\sum_{k \geq 1}\left(a_{k}(v) \cos k \theta+b_{k}(v) \sin k \theta\right) \frac{R^{k}}{r^{k}} .
$$

This satisfies the announced statement.

3. It suffices to check that $a_{0}(v)=0$.

\subsection{First term in slow variable with the logarithmic solution}

We are in the situation where the boundary value problem (2.3) defining the corrector has in general no solution. To circumvent this obstruction, the logarithmic solution has to be considered. However, it tends to infinity at infinity, it is not of finite energy in $\mathbb{R}^{2} \backslash \bar{\omega}$ and has to be considered only in $\Omega_{0}$. Its trace on $\partial \Omega_{0}$ is of size one and has to be corrected. That is why we introduce the function $w$ defined as the solution of

$$
\left\{\begin{aligned}
-\Delta w & =0 & & \text { in } \Omega_{0}, \\
w & =\ln |x| & & \text { on } \partial \Omega_{0} .
\end{aligned}\right.
$$

The idea is now to combine the logarithmic solution and the lifted $w$ to build a corrector incorporating the scales 1 and $\varepsilon$. To build this corrector, we search coefficients $a(\varepsilon)$ and $b(\varepsilon)$ such that $r_{\varepsilon}^{1}$ defined by

$$
u_{\varepsilon}(x)=u_{0}(x)+a(\varepsilon) \ln |x|+b(\varepsilon) w(x)+r_{\varepsilon}^{1}(x),
$$

is reduced with respect to $r_{\varepsilon}^{0}:=u_{\varepsilon}-u_{0}$. The remainder $r_{\varepsilon}^{1}$ satisfies

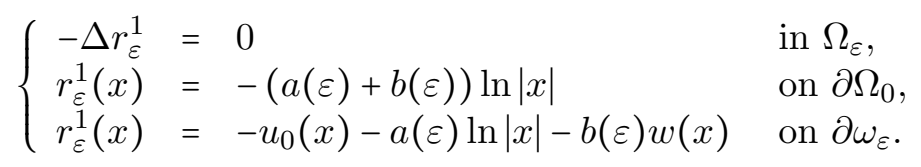


For $x \in \partial \Omega_{0}$, we have

$$
r_{\varepsilon}^{1}(x)=\mathcal{O}(1) \quad \Leftrightarrow \quad a(\varepsilon)+b(\varepsilon)=\mathcal{O}(1) .
$$

For $x \in \partial \omega_{\varepsilon}$, there exists $X \in \mathcal{B}(0,1)$ such that $x=\varepsilon X$ and we have

$$
r_{\varepsilon}^{1}(x)=\mathcal{O}(1) \quad \Leftrightarrow \quad u_{0}(0)+a(\varepsilon) \ln \varepsilon+w(0) b(\varepsilon)=\mathcal{O}(1)
$$

Hence we solve the linear system in the unknowns $(a(\varepsilon), b(\varepsilon))$ :

$$
\left\{\begin{aligned}
a(\varepsilon)+b(\varepsilon) & =0 \\
a(\varepsilon) \ln \varepsilon+b(\varepsilon) w(0) & =-u_{0}(0)
\end{aligned}\right.
$$

to set

$$
a(\varepsilon)=\frac{1}{w(0)-\ln \varepsilon} u_{0}(0) \quad \text { and } \quad b(\varepsilon)=\frac{-1}{w(0)-\ln \varepsilon} u_{0}(0) .
$$

A new scale appears:

$$
h_{\varepsilon}=\frac{1}{w(0)-\ln \varepsilon}=\frac{-1}{\ln \varepsilon}+\mathcal{O}\left(\frac{1}{\ln ^{2} \varepsilon}\right) \quad \text { as } \varepsilon \rightarrow 0 .
$$

We define the normalized corrector $\mathfrak{w}$ as

$$
\mathfrak{w}(x)=\ln |x|-w(x) .
$$

Let us remark that $\Delta \mathfrak{w}=2 \pi \delta_{0}$ in $\mathcal{D}^{\prime}\left(\Omega_{0}\right)$ and $\mathfrak{w}=0$ on $\partial \Omega_{0}$. It is a type of Green function for the domain $\Omega_{0}$. Let us emphasize that this function $\mathfrak{w}$ is defined on $\Omega_{\varepsilon}$ and can not be defined in either $\Omega_{0}$, either $\mathbb{R}^{2} \backslash \bar{\omega}$, the limit domains where the usual correctors are defined: the usual correctors in the slow variable $x$ are defined on $\Omega_{0}$ while the correctors in the rescaled variable $x / \varepsilon$ are defined in $\mathbb{R}^{2} \backslash \bar{\omega}$.

We approximate $u_{\varepsilon}$ by

$$
u_{\varepsilon}(x)=u_{0}(x)+u_{0}(0) \mathfrak{w}(x) h_{\varepsilon}+\tilde{r}_{\varepsilon}^{1}(x) .
$$

\subsection{Second term in fast variable}

We check that the remainder $\tilde{r}_{\varepsilon}^{1}$ satisfies

$$
\left\{\begin{aligned}
-\Delta \tilde{r}_{\varepsilon}^{1} & =0 & & \text { in } \Omega_{\varepsilon} \\
\tilde{r}_{\varepsilon}^{1} & =0 & & \text { on } \partial \Omega_{0} \\
\tilde{r}_{\varepsilon}^{1} & =-u_{0}-u_{0}(0) \mathfrak{w} h_{\varepsilon} & & \text { on } \partial \omega_{\varepsilon} .
\end{aligned}\right.
$$

The functions $u_{0}$ and $w$ are analytic in the vicinity of 0 . Hence, for $x=\varepsilon X \in \partial \omega_{\varepsilon}$, we have

$$
\begin{aligned}
\tilde{r}_{\varepsilon}^{1}(x)= & -u_{0}(0)-\sum_{k \geq 1} D^{k} u_{0}(0)[X, \ldots, X] \frac{\varepsilon^{k}}{k !} \\
& -u_{0}(0)\left[\ln \varepsilon+\ln |X|-w(0)-\sum_{k \geq 1} D^{k} w(0)[X, \ldots, X] \frac{\varepsilon^{k}}{k !}\right] h_{\varepsilon} \\
= & -\sum_{k \geq 1} D^{k} u_{0}(0)[X, \ldots, X] \frac{\varepsilon^{k}}{k !}-u_{0}(0) \ln |X| h_{\varepsilon}+u_{0}(0) \sum_{k \geq 1} D^{k} w(0)[X, \ldots, X] h_{\varepsilon} \frac{\varepsilon^{k}}{k !} .
\end{aligned}
$$

There are two kinds of terms: 
- the terms coming from Taylor expansions at the origin 0 of functions $\left(u_{0}, w\right)$ defined as solution of a Laplace boundary value problem posed in $\Omega_{0}$,

- the term in $\ln |X|$ which provides information about the geometry of the inclusion $\omega$.

All these terms are zero mean value on $\partial \omega_{\varepsilon}$. They can be lifted by using Lemma 2.1.

Since we made the assumption that $\omega$ is a unit ball $\mathcal{B}(0,1)$, the term $\ln |X|$ cancels. Thus the remainder $\tilde{r}_{\varepsilon}^{1}$ reads for $x=\varepsilon X \in \partial \omega_{\varepsilon}$ :

$$
\tilde{r}_{\varepsilon}^{1}(x)=\sum_{k \geq 1} u_{k}(X) \varepsilon^{k}+\sum_{k \geq 1} w_{k}(X) h_{\varepsilon} \varepsilon^{k}
$$

with

$$
u_{k}=-\frac{1}{k !} D^{k} u_{0}(0)[X, \ldots, X] \quad \text { and } \quad w_{k}=\frac{u_{0}(0)}{k !} D^{k} w(0)[X, \ldots, X] .
$$

Each appearing term in the remainder $\tilde{r}_{\varepsilon}^{1}$ is a homogeneous harmonic polynomial. Hence the mean value property insures that $\int_{\partial \mathcal{B}(0,1)} u_{k}=0$ and $\int_{\partial \mathcal{B}(0,1)} w_{k}=0$. Then Lemma 2.1 (point 3) can be applied. We set $U_{k, 1}$ and $W_{k, 1}$ the solution of problem (2.4) with $v=u_{k}$ and $v=w_{k}$ respectively. These functions can be decomposed as

$$
\begin{aligned}
U_{k, 1}(X) & =\sum_{j=1}^{k} U_{k, 1}^{j}(X) \quad \text { with } \quad U_{k, 1}^{j}(r, \theta)=\frac{a_{j}\left(u_{k}\right) \cos j \theta+b_{j}\left(u_{k}\right) \sin j \theta}{r^{j}}, \\
W_{k, 1}(X) & =\sum_{j=1}^{k} W_{k, 1}^{j}(X) \quad \text { with } \quad W_{k, 1}^{j}(r, \theta)=\frac{a_{j}\left(w_{k}\right) \cos j \theta+b_{j}\left(w_{k}\right) \sin j \theta}{r^{j}} .
\end{aligned}
$$

We then approximate $u_{\varepsilon}$ by

$$
u_{\varepsilon}(x)=u_{0}(x)+u_{0}(0) \mathfrak{w}(x) h_{\varepsilon}+\sum_{k \geq 1} U_{k, 1}\left(\frac{x}{\varepsilon}\right) \varepsilon^{k}+\sum_{k \geq 1} W_{k, 1}\left(\frac{x}{\varepsilon}\right) h_{\varepsilon} \varepsilon^{k}+\tilde{r}_{\varepsilon}^{2}(x) .
$$

\subsection{Third term in slow variable}

We have now to study the remainder $\tilde{r}_{\varepsilon}^{2}$. It satisfies

$$
\left\{\begin{aligned}
-\Delta \tilde{r}_{\varepsilon}^{2} & =0 & & \text { in } \Omega_{\varepsilon}, \\
\tilde{r}_{\varepsilon}^{2} & =-\sum_{k \geq 1} U_{k, 1}\left(\frac{x}{\varepsilon}\right) \varepsilon^{k}-\sum_{k \geq 1} W_{k, 1}\left(\frac{x}{\varepsilon}\right) h_{\varepsilon} \varepsilon^{k} & & \text { on } \partial \Omega_{0}, \\
\tilde{r}_{\varepsilon}^{2} & =0 & & \text { on } \partial \omega_{\varepsilon} .
\end{aligned}\right.
$$

According to decomposition (2.8) and (2.9), the traces $T U_{k, 1}^{j}$ and $T W_{k, 1}^{j}$ on $\partial \Omega_{0}$ of the functions $U_{k, 1}^{j}\left(\frac{x}{\varepsilon}\right)$ and $V_{k, 1}^{j}\left(\frac{x}{\varepsilon}\right)$ satisfy

$$
\left\|T U_{k, 1}^{j}\right\|_{\mathrm{H}^{1 / 2}\left(\partial \Omega_{0}\right)}=\mathcal{O}\left(\varepsilon^{j}\right) \quad \text { and } \quad\left\|T W_{k, 1}^{j}\right\|_{\mathrm{H}^{1 / 2}\left(\partial \Omega_{0}\right)}=\mathcal{O}\left(\varepsilon^{j}\right) .
$$

Then, we get

$$
\left\|T U_{k, 1}\right\|_{\mathrm{H}^{1 / 2}\left(\partial \Omega_{0}\right)}=\mathcal{O}(\varepsilon) \quad \text { and } \quad\left\|T W_{k, 1}\right\|_{\mathrm{H}^{1 / 2}\left(\partial \Omega_{0}\right)}=\mathcal{O}(\varepsilon) .
$$

We now lift these functions in slow variables and define $u_{k, 1}$ and $w_{k, 1}$ as the solution of the normalized problems:

$$
\left\{\begin{array} { r l r l } 
{ - \Delta u _ { k , 1 } } & { = 0 } & { } & { \text { in } \Omega _ { 0 } , } \\
{ u _ { k , 1 } } & { = \frac { - 1 } { \varepsilon } T U _ { k , 1 } } & { } & { \text { on } \partial \Omega _ { 0 } , }
\end{array} \quad \text { and } \quad \left\{\begin{array}{rlrl}
-\Delta w_{k, 1} & =0 & & \text { in } \Omega_{0}, \\
w_{k, 1} & =\frac{-1}{\varepsilon} T W_{k, 1} & & \text { on } \partial \Omega_{0} .
\end{array}\right.\right.
$$


We can read $u_{\varepsilon}$ :

$$
\begin{aligned}
u_{\varepsilon}(x)=u_{0}(x)+h_{\varepsilon} u_{0}(0) \mathfrak{w}(x)+\sum_{k \geq 1} U_{k, 1}\left(\frac{x}{\varepsilon}\right) \varepsilon^{k}+\sum_{k \geq 1} W_{k, 1}\left(\frac{x}{\varepsilon}\right) h_{\varepsilon} \varepsilon^{k} & \\
& +\sum_{k \geq 2} u_{k-1,1}(x) \varepsilon^{k}+\sum_{k \geq 2} w_{k-1,1}(x) h_{\varepsilon} \varepsilon^{k}+\tilde{r}_{\varepsilon}^{3}(x) .
\end{aligned}
$$

\subsection{Antsatz of asymptotic expansion}

We inject the remainder $\tilde{r}_{\varepsilon}^{3}$ in the equation and obtain:

$$
\left\{\begin{aligned}
-\Delta \tilde{r}_{\varepsilon}^{3} & =0 & & \text { in } \Omega_{\varepsilon} \\
\tilde{r}_{\varepsilon}^{3} & =0 & & \text { on } \partial \Omega_{0} \\
\tilde{r}_{\varepsilon}^{3} & =-\sum_{k \geq 2} u_{k-1,1} \varepsilon^{k}-\sum_{k \geq 2} w_{k-1,1} h_{\varepsilon} \varepsilon^{k} & & \text { on } \partial \omega_{\varepsilon} .
\end{aligned}\right.
$$

The function $u_{k, 1}$ and $w_{k, 1}$ have a value at 0 which is a priori not 0 . This suggests to mimic the approach already used to start the asymptotics and to define $\tilde{r}_{\varepsilon}^{4}$ by:

$$
\begin{aligned}
u_{\varepsilon}(x)= & u_{0}(x)+u_{0}(0) \mathfrak{w}(x) h_{\varepsilon}+\sum_{k \geq 1} U_{k, 1}\left(\frac{x}{\varepsilon}\right) \varepsilon^{k}+\sum_{k \geq 1} W_{k, 1}\left(\frac{x}{\varepsilon}\right) h_{\varepsilon} \varepsilon^{k} \\
& +\sum_{k \geq 2} u_{k-1,1}(x) \varepsilon^{k}+\sum_{k \geq 2} w_{k-1,1}(x) h_{\varepsilon} \varepsilon^{k} \\
& +\sum_{k \geq 2} u_{k-1,1}(0) \mathfrak{w}(x) h_{\varepsilon} \varepsilon^{k}+\sum_{k \geq 2} w_{k-1,1}(0) \mathfrak{w}(x) h_{\varepsilon}^{2} \varepsilon^{k}+\tilde{r}_{\varepsilon}^{4}(x) .
\end{aligned}
$$

This justifies the antsatz

$$
\begin{aligned}
u_{\varepsilon}(x)=u_{0}(x)+u_{0}(0) \mathfrak{w}(x) h_{\varepsilon}+\sum_{j \geq 1, k \geq 2} & \alpha_{j, k} \mathfrak{w}(x) h_{\varepsilon}^{j} \varepsilon^{k} \\
& +\sum_{j \geq 0, k \geq 1} V_{j, k}\left(\frac{x}{\varepsilon}\right) h_{\varepsilon}^{j} \varepsilon^{k}+\sum_{j \geq 0, k \geq 2} v_{j, k}(x) h_{\varepsilon}^{j} \varepsilon^{k},
\end{aligned}
$$

where any coefficient $\alpha_{j, k}$ and any functions $V_{j, k}$ and $v_{j, k}$ are obtained by reiterating the previous steps.

\subsection{Justification of the order of convergence}

Now we have to justify this formal expansion by using an a priori error estimate. Let us introduce the remainder of the asymptotic expansion truncated after the order $h_{\varepsilon}^{J} \varepsilon^{K}$

$$
\begin{aligned}
r_{\varepsilon}^{J, K}(x)=u_{\varepsilon}(x)-u_{0}(x)-u_{0}(0) \mathfrak{w}(x) & h_{\varepsilon}-\sum_{(j, k) \in \mathcal{K}_{J, K}, j \geq 1, k \geq 2} \alpha_{j, k} \mathfrak{w}(x) h_{\varepsilon}^{j} \varepsilon^{k} \\
& +\sum_{(j, k) \in \mathcal{K}_{J, K}, k \geq 1} V_{j, k}\left(\frac{x}{\varepsilon}\right) h_{\varepsilon}^{j} \varepsilon^{k}+\sum_{(j, k) \in \mathcal{K}_{J, K}, k \geq 2} v_{j, k}(x) h_{\varepsilon}^{j} \varepsilon^{k},
\end{aligned}
$$

with

$$
\mathcal{K}_{J, K}=\left\{(j, k) \in \mathbb{N}^{2} ; k<K \text { or } k=K \text { and } j \leq J\right\} .
$$

By construction, $r_{\varepsilon}^{J, K}$ is harmonic. 
By the definition of the trace space of $\mathrm{H}^{1}\left(\Omega_{\varepsilon}\right)$ with the norm

$$
\|f\|_{T \mathrm{H}^{1}\left(\Omega_{\varepsilon}\right)}=\inf \left\{\|u\|_{\mathrm{H}^{1}\left(\Omega_{\varepsilon}\right)} ; u \in \mathrm{H}^{1}\left(\Omega_{\varepsilon}\right) \text { with } u=f \text { on } \partial \Omega_{\varepsilon}\right\},
$$

we have

$$
\left\|r_{\varepsilon}^{J, K}\right\|_{\mathrm{H}^{1}\left(\Omega_{\varepsilon}\right)}=\left\|r_{\varepsilon}^{J, K}\right\|_{T \mathrm{H}^{1}\left(\Omega_{\varepsilon}\right)} .
$$

The equality holds when $u$ is harmonic since its achieves the infimum in the definition of the $T \mathrm{H}^{1}$ norm. To overcome the difficulty to evaluate the $T \mathrm{H}^{1}$ norm of a given function, we also define the intrinsic Sobolev space $\mathrm{H}^{1 / 2}$ of the boundary of an $\varepsilon$-dependent domain $\Omega_{\varepsilon}$ as a subspace of $\mathrm{L}^{2}\left(\partial \Omega_{\varepsilon}\right)$ with finite norm

$$
\|f\|_{\mathrm{H}^{1 / 2}\left(\partial \Omega_{\varepsilon}\right)}=\|f\|_{\mathrm{L}^{2}\left(\partial \Omega_{\varepsilon}\right)}+[f]_{2, \partial \Omega_{\varepsilon}},
$$

with

$$
[f]_{2, \partial \Omega_{\varepsilon}}^{2}=\iint_{\partial \Omega_{\varepsilon} \times \partial \Omega_{\varepsilon}} \frac{|f(x)-f(y)|^{2}}{|x-y|^{2}} d \sigma_{x} d \sigma_{y} .
$$

E. Gagliardo has shown in [7] that, if the domain is Lipschitz, the two different norms on $\mathrm{H}^{1 / 2}$ are equivalent. For a familly of domains parametrized by $\varepsilon$, this means that the domains should be uniformly Lipschitz with respect to $\varepsilon$. In the case we are studying: a single interior perturbation $\Omega_{\varepsilon}=\Omega_{0} \backslash \overline{\omega_{\varepsilon}}$ where the nucleation center 0 belongs to $\Omega_{0}$, the uniform Lipschitz property is not satisfied and the norms $\mathrm{H}^{1 / 2}$ and $T \mathrm{H}^{1}$ may be non equivalent.

V. Maz'ya and S. Poborchi discuss in [17, Section 4.1.3] situations where this property is violated. The two terms in the intrinsic norm should be weighted. We quote their result once adapted to the space $\mathrm{H}^{1 / 2}$ in the case of plane domains: the trace norm $\|f\|_{T \mathrm{H}^{1}\left(\Omega_{\varepsilon}\right)}$ is equivalent, uniformly in $\varepsilon$, to the norm

$$
(\varepsilon|\ln \varepsilon|)^{-1 / 2}\|f\|_{L^{2}\left(\partial \Omega_{\varepsilon}\right)}+[f]_{2, \partial \Omega_{\varepsilon}} .
$$

We have now to face the question of evaluating on $\partial \omega \varepsilon$ various norm of the trace of a function $f$ wich is smooth around 0. Quoting [4, Lemma 3.2], if $f$ is a smooth function defined around 0 , let $M \geq 0$ be such that for all multi-indices $k$ with $|k|<M, \partial_{k} f(0)=0$. Then,

$$
\begin{aligned}
\|f\|_{\mathrm{H}^{1 / 2}(\varepsilon \partial \omega)} & \leq C \varepsilon^{M}, \\
\|f\|_{T \mathrm{H}^{1}(\varepsilon \omega)} & \leq C|\ln \varepsilon|^{-1 / 2} \varepsilon^{M-1 / 2},
\end{aligned}
$$

with $C$ independent of $\varepsilon$. Applying this to $r_{\varepsilon}^{J, K}$, we get

$$
\left\|r_{\varepsilon}^{J, K}\right\|_{\mathrm{H}^{1}\left(\Omega_{\varepsilon}\right)} \leq C h_{\varepsilon}^{J-1 / 2} \varepsilon^{K-1 / 2} .
$$

Remark 2.2 Let us conclude this section by noting that the usual a priori estimates can be applied for the second remainder $r_{\varepsilon}^{2}$ directly since its trace on the non-uniformly Lipschitz boundary $\partial \omega_{\varepsilon}$ is 0 . Hence, we get directly from $(2.10)-(2.11)$ that $\left\|r_{\varepsilon}^{2}\right\|_{\mathrm{H}^{1}\left(\Omega_{\varepsilon}\right)} \leq C \varepsilon^{2}$, that is:

$$
u_{\varepsilon}(x)=u_{0}(x)+u_{0}(0) \mathfrak{w}(x) h_{\varepsilon}+U_{1,1}\left(\frac{x}{\varepsilon}\right) \varepsilon+W_{1,1}\left(\frac{x}{\varepsilon}\right) h_{\varepsilon} \varepsilon+\mathcal{O}_{\mathrm{H}^{1}\left(\Omega_{\varepsilon}\right)}\left(\varepsilon^{2}\right) .
$$




\subsection{Numerical illustration}

In this subsection, we illustrate the contribution of the first two terms of the expansion (2.12). For this, we choose for $\Omega_{0}$ the rectangle $[-1,1] \times[0,1]$ and we consider the

$$
f(x)=2\left(x_{2}^{2}-x_{2}\right)+2\left(x_{1}^{2}-1\right) .
$$

Then the solution $u_{0}$ of the problem (2.2) is given explicitely by

$$
u_{0}(x)=-\left(x_{1}^{2}-1\right)\left(x_{2}^{2}-x_{2}\right),
$$

as illustrated in Figure 1.

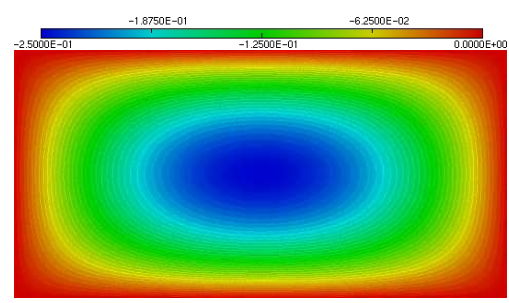

Figure 1: $u_{0}$

For numerical computation, we take $\varepsilon=1 / 1.5^{7} \simeq 0.0585$. With this parameter, we have

$$
h_{\varepsilon} \simeq-0.100 \quad \text { and } \quad \frac{-1}{\ln \varepsilon} \simeq-0.088 .
$$

We compute the solution $u_{\varepsilon}$ of problem (2.1) by a finite element method using the Finite Element Library MÉLINA (see [12]). The computed solution is given in Figure 2. The

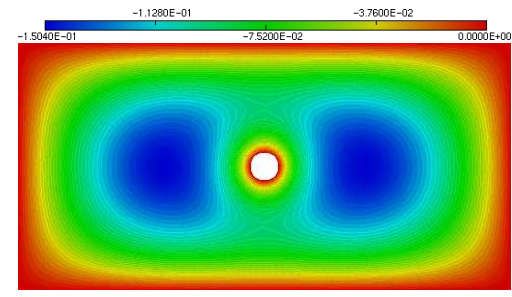

Figure 2: $u_{\varepsilon}$

difference $r_{\varepsilon}^{0}=u_{\varepsilon}-u_{0}$ is given in Figure 3. We observe that that error is located around the inclusion since $u_{0}$ does not satisfy the Dirichlet condition on the inclusion.

Let us know observe the effect of the second term of the asymptotics. We compute an approximation of the function $w$, solution of $(2.5)$ on $\Omega_{0}$ by a finite element method, see Figure $4(\mathrm{a})$. The second term $x \mapsto u_{0}(0) \mathfrak{w}(w) h_{\varepsilon}=u_{0}(0)(\ln |x|-w(x)) h_{\varepsilon}$ of the expansion is computed in Figure 4(b). We add the first two terms of the expansion in Figure 5(a) with the scale $h_{\varepsilon}$. We then compute the second remainder $\tilde{r}_{\varepsilon}^{1}=u_{\varepsilon}-u_{0}-u_{0}(0) \mathfrak{w} h_{\varepsilon}$ in Figure 5(b). We observe the error is now located between at the middle of $\Omega_{\varepsilon}$ far away the boundary.

If we replace the asymptotics scale $h_{\varepsilon}$ by its first order expansion $-1 / \ln \varepsilon$, we change the second term of the expansion by the multiplicative term as illustrated in Figure 4(c). 


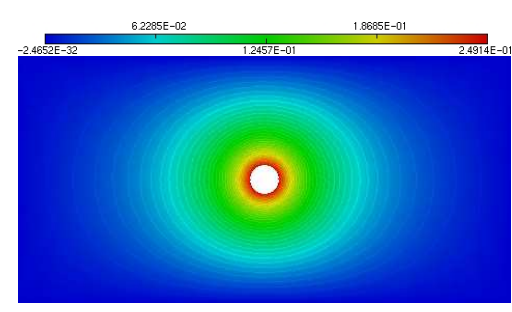

Figure 3: First order approximation $u_{\varepsilon}-u_{0}$

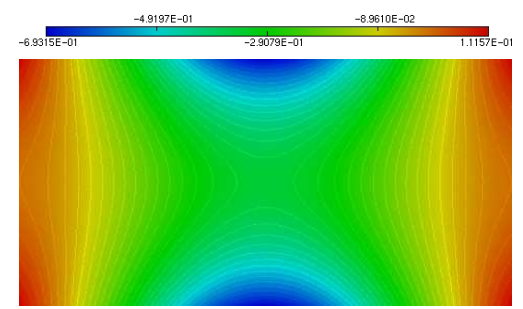

(a) $w$

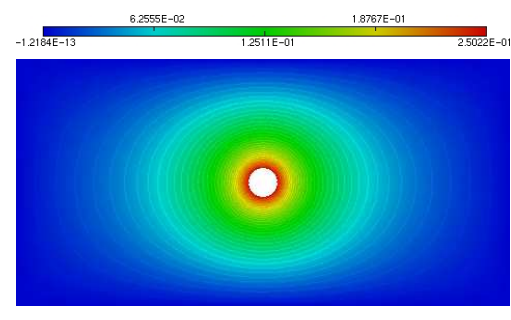

(b) $u_{0}(0) \mathfrak{w} h_{\varepsilon}$

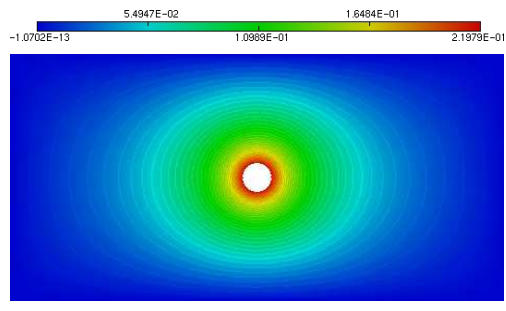

(c) $-u_{0}(0) \mathfrak{w} / \ln \varepsilon$

Figure 4: Second term of the expansion

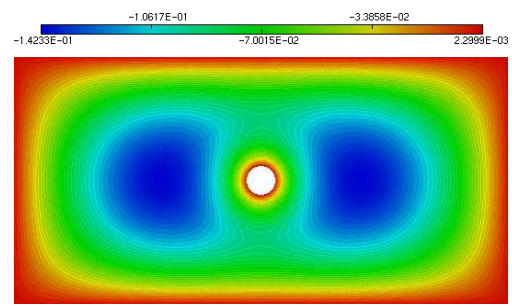

(a) $u_{0}+u_{0}(0) \mathfrak{w} h_{\varepsilon}$

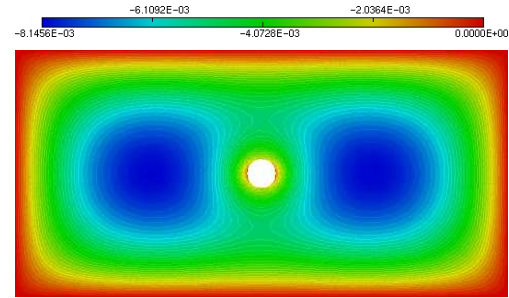

(b) $u_{\varepsilon}-u_{0}-u_{0}(0) \mathfrak{w} h_{\varepsilon}$

Figure 5: Second order approximation $u_{0}+u_{0}(0) \mathfrak{w} h_{\varepsilon}$

If we approximate $h_{\varepsilon}$ and $-1 / \ln \varepsilon$ and then $u_{\varepsilon}$ by $u_{0}-u_{0}(0) \mathfrak{w} \ln \varepsilon$ as in Figures $6(\mathrm{a})$ and $6(\mathrm{~b})$, we observe that the error if located around the inclusion. We highlight the interest of choosing the adapted scale $h_{\varepsilon}$ and not its first order approximation $-1 / \ln \varepsilon$.

Table 1 gives the error norms when we aprroximate $u_{\varepsilon}$ by the first terms of its expansion. 


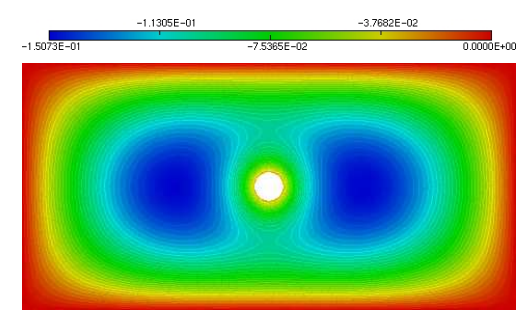

(a) $u_{0}-u_{0}(0) \mathfrak{w} / \ln \varepsilon$

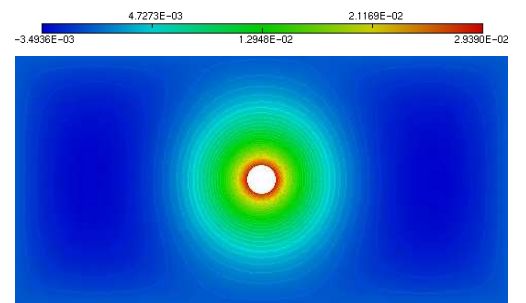

(b) $u_{\varepsilon}-u_{0}+u_{0}(0) \mathfrak{w} / \ln \varepsilon$

Figure 6: Second order approximation $u_{0}-u_{0}(0) \mathfrak{w} / \ln \varepsilon$

\begin{tabular}{|c|c|c|c|}
\cline { 2 - 4 } \multicolumn{1}{c|}{} & $u_{\varepsilon}-u_{0}$ & $u_{\varepsilon}-u_{0}-u_{0}(0) \mathfrak{w} h_{\varepsilon}$ & $u_{\varepsilon}-u_{0}+u_{0}(0) \mathfrak{w} / \ln \varepsilon$ \\
\hline $\mathrm{L}^{2}$-norm & $7.799 \mathrm{e}-2$ & $6.88 \mathrm{e}-3$ & $6.56 \mathrm{e}-3$ \\
\hline $\mathrm{H}^{1}$-norm & $4.0277 \mathrm{e}-1$ & $2.865 \mathrm{e}-2$ & $5.290 \mathrm{e}-2$ \\
\hline
\end{tabular}

Table 1: Error norms

\section{Two separated inclusions case}

Now fix two distinct points $A$ et $B$ in $\Omega$ and set $\mathbf{d}=\overrightarrow{A B}$. We consider the Dirichlet boundary value problem:

$$
\left\{\begin{array}{rllll}
-\Delta u_{\varepsilon} & = & f & \text { in } & \Omega_{\varepsilon} \\
u_{\varepsilon} & = & 0 & \text { on } & \partial \Omega_{\varepsilon}
\end{array}\right.
$$

set in the domain:

$$
\Omega_{\varepsilon}=\Omega_{0} \backslash\left(\overline{\omega_{\varepsilon}^{A} \cup \omega_{\varepsilon}^{B}}\right), \quad \text { with } \quad \omega_{\varepsilon}^{A}=\mathcal{B}(A, \varepsilon) \quad \text { and } \quad \omega_{\varepsilon}^{B}=\mathcal{B}(B, \varepsilon),
$$

as illustrated in Figure 7. Introduce the functions $w_{A}$ and $w_{B}$ solutions of

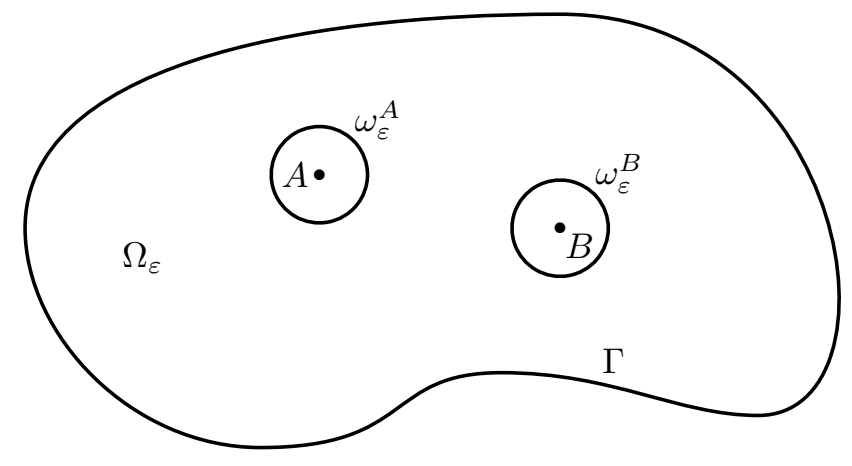

Figure 7: The perturbed domain

$$
\left\{\begin{array} { r l r l } 
{ - \Delta w _ { A } } & { = 0 } & { } & { \text { in } \Omega _ { 0 } , } \\
{ w _ { A } } & { = \operatorname { l n } | x - A | } & { } & { \text { on } \partial \Omega _ { 0 } , }
\end{array} \text { and } \left\{\begin{array}{rlrl}
-\Delta w_{B} & =0 & & \text { in } \Omega_{0}, \\
w_{B} & =\ln |x-B| & & \text { on } \partial \Omega_{0} .
\end{array}\right.\right.
$$

We follow the previously introduced algorithm and seek coefficients $a_{A}(\varepsilon), b_{A}(\varepsilon)$ and $a_{B}(\varepsilon), b_{B}(\varepsilon)$ so that the remainder $r_{\varepsilon}^{1}$ defined as

$$
u_{\varepsilon}(x)=u_{0}(x)+a_{A}(\varepsilon) \ln |x-A|+b_{A}(\varepsilon) w_{A}(x)+a_{B}(\varepsilon) \ln |x-B|+b_{B}(\varepsilon) w_{B}(x)+r_{\varepsilon}^{1}(x),
$$


is smaller than $r_{\varepsilon}^{0}=u_{\varepsilon}-u_{0}$. The function $r_{\varepsilon}^{1}$ satisfies

$$
\left\{\begin{array}{rlrl}
-\Delta r_{\varepsilon}^{1}= & 0 & & \text { in } \Omega_{\varepsilon}, \\
r_{\varepsilon}^{1}(x) & =-\left(a_{A}(\varepsilon)+b_{A}(\varepsilon)\right) \ln |x-A|-\left(a_{B}(\varepsilon)+b_{B}(\varepsilon)\right) \ln |x-B| & & \text { on } \partial \Omega_{0}, \\
r_{\varepsilon}^{1}(x) & =-u_{0}(x)-a_{A}(\varepsilon) \ln |x-A|-b_{A}(\varepsilon) w_{A}(x) & & \\
-a_{B}(\varepsilon) \ln |x-B|-b_{B}(\varepsilon) w_{B}(x) & & \text { on } \partial\left(\omega_{\varepsilon}^{A} \cup \omega_{\varepsilon}^{B}\right) .
\end{array}\right.
$$

The functions $x \mapsto \ln |x-A|$ and $x \mapsto \ln |x-B|$ defined on $\partial \Omega_{0}$ are linearly independent since $A \neq B$. Then for $x \in \partial \Omega_{0}$,

$$
r_{\varepsilon}^{1}(x)=\mathcal{O}(1) \quad \Leftrightarrow \quad a_{A}(\varepsilon)+b_{A}(\varepsilon)=\mathcal{O}(1) \quad \text { and } \quad a_{B}(\varepsilon)+b_{B}(\varepsilon)=\mathcal{O}(1) .
$$

For $x \in \partial \omega_{\varepsilon}^{A}$, there exists $X \in \mathcal{B}(0,1)$ such that $x=A+\varepsilon X$ and we have

$$
r_{\varepsilon}^{1}(x)=\mathcal{O}(1) \Leftrightarrow u_{0}(A)+a_{A}(\varepsilon) \ln \varepsilon+b_{A}(\varepsilon) w_{A}(A)+a_{B}(\varepsilon) \ln |d|+b_{B}(\varepsilon) w_{B}(A)=\mathcal{o}(1) .
$$

By symmetry, for $x \in \partial \omega_{\varepsilon}^{B}$, there exists $X \in \mathcal{B}(0,1)$ such that $x=B+\varepsilon X$ and we have

$$
r_{\varepsilon}^{1}(x)=\mathcal{O}(1) \Leftrightarrow u_{0}(B)+a_{A}(\varepsilon) \ln |d|+b_{A}(\varepsilon) w_{A}(B)+a_{B}(\varepsilon) \ln \varepsilon+b_{B}(\varepsilon) w_{B}(B)=\mathcal{o}(1) .
$$

Hence we solve the linear system in the unknowns $\left(a_{A}(\varepsilon), b_{A}(\varepsilon), a_{B}(\varepsilon), b_{B}(\varepsilon)\right)$ :

$$
\left\{\begin{aligned}
a_{A}(\varepsilon)+b_{A}(\varepsilon) & =0, \\
a_{B}(\varepsilon)+b_{B}(\varepsilon) & =0, \\
a_{A}(\varepsilon) \ln \varepsilon+b_{A}(\varepsilon) w_{A}(A)+a_{B}(\varepsilon) \ln |d|+b_{B}(\varepsilon) w_{B}(A) & =-u_{0}(A), \\
a_{A}(\varepsilon) \ln |d|+b_{A}(\varepsilon) w_{A}(B)+a_{B}(\varepsilon) \ln \varepsilon+b_{B}(\varepsilon) w_{B}(B) & =-u_{0}(B) .
\end{aligned}\right.
$$

Writing $b_{A}(\varepsilon)=-a_{A}(\varepsilon)$ and $b_{B}(\varepsilon)=-a_{B}(\varepsilon)$, the system can be reduced to

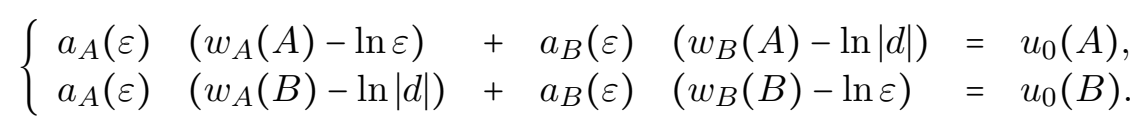

We set

$$
\delta(\varepsilon)=\left(w_{A}(B)-\ln |d|\right)\left(w_{B}(A)-\ln |d|\right)-\left(w_{A}(A)-\ln \varepsilon\right)\left(w_{B}(B)-\ln \varepsilon\right)
$$

and get:

$$
\left\{\begin{array}{l}
a_{A}(\varepsilon)=-b_{A}(\varepsilon)=\frac{\ln \varepsilon-w_{B}(B)}{\delta(\varepsilon)} u_{0}(A)+\frac{w_{B}(A)-\ln |d|}{\delta(\varepsilon)} u_{0}(B), \\
a_{B}(\varepsilon)=-b_{B}(\varepsilon)=\frac{w_{A}(B)-\ln |d|}{\delta(\varepsilon)} u_{0}(A)+\frac{\ln \varepsilon-w_{A}(A)}{\delta(\varepsilon)} u_{0}(B) .
\end{array}\right.
$$

We check that the leading terms are the same as the ones obtained by considering a single inclusion at $A$ and a single inclusion at $B$ :

$$
a_{A}(\varepsilon)=\frac{-1}{\ln \varepsilon} u_{0}(A)+\mathcal{O}\left(\frac{1}{\ln ^{2} \varepsilon}\right) \quad \text { and } \quad a_{B}(\varepsilon)=\frac{-1}{\ln \varepsilon} u_{0}(B)+\mathcal{O}\left(\frac{1}{\ln ^{2} \varepsilon}\right) \text { as } \varepsilon \rightarrow 0 .
$$

The presence of two defects appears in $a_{A}(\varepsilon)$ and in $a_{B}(\varepsilon)$ but only in higher order term in the asymptotic expansion of the coefficients. Setting

$$
\mathfrak{w}_{A}(x)=\ln |x-A|-w_{A}(x) \quad \text { and } \quad \mathfrak{w}_{B}(x)=\ln |x-B|-w_{B}(x) .
$$


We follow the algorithmic method of Section 2 and approximate $u_{\varepsilon}$ by

$$
u_{\varepsilon}=u_{0}+a_{A}(\varepsilon) \mathfrak{w}_{A}+a_{B}(\varepsilon) \mathfrak{w}_{B}+\tilde{r}_{\varepsilon}^{1} .
$$

Then, we check that $\tilde{r}_{\varepsilon}^{1}$ satisfies

$$
\left\{\begin{aligned}
-\Delta \tilde{r}_{\varepsilon}^{1} & =0 & & \text { in } \Omega_{\varepsilon} \\
\tilde{r}_{\varepsilon}^{1} & =0 & & \text { on } \partial \Omega_{0} \\
\tilde{r}_{\varepsilon}^{1} & =-u_{0}-a_{A}(\varepsilon) \mathfrak{w}_{A}-a_{B}(\varepsilon) \mathfrak{w}_{B} & & \text { on } \partial\left(\omega_{\varepsilon}^{A} \cup \omega_{\varepsilon}^{B}\right)
\end{aligned}\right.
$$

For $x=A+\varepsilon X \in \partial \omega_{\varepsilon}^{A}$, we have

$$
|x-B|^{2}=|A-B+\varepsilon X|^{2}=|\mathbf{d}|^{2}-2 \varepsilon\langle\mathbf{d}, X\rangle+\varepsilon^{2},
$$

then

$$
\ln |x-B|=\ln |\mathbf{d}|+\sum_{k \geq 1} b_{k}(X) \varepsilon^{k} .
$$

Consequently, the remainder $\tilde{r}_{\varepsilon}^{1}$ reads for $x=A+\varepsilon X \in \partial \omega_{\varepsilon}^{A}$

$$
\begin{aligned}
\tilde{r}_{\varepsilon}^{1}(x)= & -u_{0}(A)-\sum_{k \geq 1} D^{k} u_{0}(A)[X, \ldots, X] \frac{\varepsilon^{k}}{k !} \\
& -a_{A}(\varepsilon)\left[\ln \varepsilon+\ln |X|-w_{A}(A)-\sum_{k \geq 1} D^{k} w_{A}(A)[X, \ldots, X] \frac{\varepsilon^{k}}{k !}\right] \\
& -a_{B}(\varepsilon)\left[\ln |\mathbf{d}|+\sum_{k \geq 1} b_{k}(X) \varepsilon^{k}-w_{B}(A)-\sum_{k \geq 1} D^{k} w_{B}(A)[X, \ldots, X] \frac{\varepsilon^{k}}{k !}\right] .
\end{aligned}
$$

Using (3.5) and the fact that $X \in \mathcal{B}(0,1)$, we check that the terms of low order cancel and thus, for $x \in \partial \omega_{\varepsilon}^{A}$,

$$
\begin{aligned}
\tilde{r}_{\varepsilon}^{1}(x)= & -\sum_{k \geq 1} D^{k} u_{0}(A)[X, \ldots, X] \frac{\varepsilon^{k}}{k !}+a_{A}(\varepsilon) \sum_{k \geq 1} D^{k} w_{A}(A)[X, \ldots, X] \frac{\varepsilon^{k}}{k !} \\
& -a_{B}(\varepsilon) \sum_{k \geq 1}\left[b_{k}(X)-\frac{1}{k !} D^{k} w_{B}(A)[X, \ldots, X]\right] \varepsilon^{k} .
\end{aligned}
$$

We rewrite the remainder on $\partial \omega_{\varepsilon}^{A}$ as

$$
\tilde{r}_{\varepsilon}^{1}(x)=\sum_{k \geq 1} u_{k}^{A}(X) \varepsilon^{k}+a_{A}(\varepsilon) \sum_{k \geq 1} v_{k}^{A}(X) \varepsilon^{k}+a_{B}(\varepsilon) \sum_{k \geq 1} w_{k}^{A B}(X) \varepsilon^{k} .
$$

By symmetry, one checks that for $x=B+\varepsilon X \in \partial \omega_{\varepsilon}^{B}$, it holds

$$
|x-A|=|B-A+\varepsilon X|=|\mathbf{d}|^{2}+2 \varepsilon\langle\mathbf{d}, X\rangle+\varepsilon^{2},
$$

then

$$
\ln |x-A|=\ln |\mathbf{d}|+\sum_{k \geq 1} a_{k}(X) \varepsilon^{k},
$$

and for $x \in \partial \omega_{\varepsilon}^{B}$,

$$
\begin{aligned}
\tilde{r}_{\varepsilon}^{1}(x)= & -\sum_{k \geq 1} D^{k} u_{0}(B)[X, \ldots, X] \frac{\varepsilon^{k}}{k !}+a_{B}(\varepsilon) \sum_{k \geq 1} D^{k} w_{B}(B)[X, \ldots, X] \frac{\varepsilon^{k}}{k !} \\
& -a_{A}(\varepsilon) \sum_{k \geq 1}\left[a_{k}(X)-\frac{1}{k !} D^{k} w_{A}(B)[X, \ldots, X]\right] \varepsilon^{k} .
\end{aligned}
$$


We can also rewrite the remainder for $x=B+\varepsilon X \in \partial \omega_{\varepsilon}^{B}$ as

$$
\tilde{r}_{\varepsilon}^{1}(x)=\sum_{k \geq 1} u_{k}^{B}(X) \varepsilon^{k}+a_{B}(\varepsilon) \sum_{k \geq 1} v_{k}^{B}(X) \varepsilon^{k}+a_{A}(\varepsilon) \sum_{k \geq 1} w_{k}^{B A}(X) \varepsilon^{k} .
$$

As in the case of one inclusion in Subsection 2.3, we lift each term appearing in the boundary of the inclusions. Thus we approximate $u_{\varepsilon}$ by

$$
\begin{aligned}
u_{\varepsilon}(x)=u_{0}(x)+ & a_{A}(\varepsilon) \mathfrak{w}_{A}(x)+a_{B}(\varepsilon) \mathfrak{w}_{B}(x) \\
+\sum_{k \geq 1} & \left(U_{k, 1}^{A}\left(\frac{x-A}{\varepsilon}\right) \varepsilon^{k}+V_{k, 1}^{A}\left(\frac{x-A}{\varepsilon}\right) a_{A}(\varepsilon) \varepsilon^{k}+W_{k, 1}^{A B}\left(\frac{x-A}{\varepsilon}\right) a_{B}(\varepsilon) \varepsilon^{k}\right) \\
& +\sum_{k \geq 1}\left(U_{k, 1}^{B}\left(\frac{x-B}{\varepsilon}\right) \varepsilon^{k}+V_{k, 1}^{B}\left(\frac{x-B}{\varepsilon}\right) a_{B}(\varepsilon) \varepsilon^{k}+W_{k, 1}^{B A}\left(\frac{x-B}{\varepsilon}\right) a_{A}(\varepsilon) \varepsilon^{k}\right)+\tilde{r}_{\varepsilon}^{2} .
\end{aligned}
$$

These new correctors generate traces on the boundary $\partial \Omega_{0}$, exactly as in the case of a single inclusion (see Subsection 2.4): we check that the reminder $\tilde{r}_{\varepsilon}^{2}$ is the solution of

$$
\left\{\begin{array}{rrr}
-\Delta \tilde{r}_{\varepsilon}^{2}= & 0 & \text { in } \Omega_{\varepsilon}, \\
\tilde{r}_{\varepsilon}^{2}= & -\sum_{k \geq 1}\left(U_{k, 1}^{A}\left(\frac{x-A}{\varepsilon}\right) \varepsilon^{k}-V_{k, 1}^{A}\left(\frac{x-A}{\varepsilon}\right) a_{A}(\varepsilon) \varepsilon^{k}+W_{k, 1}^{A B}\left(\frac{x-A}{\varepsilon}\right) a_{B}(\varepsilon) \varepsilon^{k}\right) & \\
& -\sum_{k \geq 1}\left(U_{k, 1}^{B}\left(\frac{x-B}{\varepsilon}\right) \varepsilon^{k}-V_{k, 1}^{B}\left(\frac{x-B}{\varepsilon}\right) a_{B}(\varepsilon) \varepsilon^{k}+W_{k, 1}^{B A}\left(\frac{x-B}{\varepsilon}\right) a_{A}(\varepsilon) \varepsilon^{k}\right) & \text { on } \partial \Omega_{0}, \\
\tilde{r}_{\varepsilon}^{2}= & 0 & \text { on } \partial\left(\omega_{\varepsilon}^{A} \cup \omega_{\varepsilon}^{B}\right) .
\end{array}\right.
$$

In particular, we get $\left\|\tilde{r}_{\varepsilon}^{2}\right\|_{\mathrm{H}^{1}\left(\Omega_{\varepsilon}\right)}=\mathcal{O}\left(\varepsilon^{2}\right)$ by the argument developed in Remark 2.2 and the order two expansion:

$$
\begin{aligned}
& u_{\varepsilon}(x)=u_{0}(x)+a_{A}(\varepsilon) \mathfrak{w}_{A}(x)+a_{B}(\varepsilon) \mathfrak{w}_{B}(x) \\
& \quad+\sum_{k \geq 1}\left(U_{k, 1}^{A}\left(\frac{x-A}{\varepsilon}\right) \varepsilon^{k}+V_{k, 1}^{A}\left(\frac{x-A}{\varepsilon}\right) a_{A}(\varepsilon) \varepsilon^{k}+W_{k, 1}^{A B}\left(\frac{x-A}{\varepsilon}\right) a_{B}(\varepsilon) \varepsilon^{k}\right) \\
& +\sum_{k \geq 1}\left(U_{k, 1}^{B}\left(\frac{x-B}{\varepsilon}\right) \varepsilon^{k}+V_{k, 1}^{B}\left(\frac{x-B}{\varepsilon}\right) a_{B}(\varepsilon) \varepsilon^{k}+W_{k, 1}^{B A}\left(\frac{x-B}{\varepsilon}\right) a_{A}(\varepsilon) \varepsilon^{k}\right)+\mathcal{O}_{\mathrm{H}^{1}\left(\Omega_{\varepsilon}\right)}\left(\varepsilon^{2}\right) .
\end{aligned}
$$

In the previous expansion many terms are of higher order than the remainder, we have shown:

Theorem 3.1 The solution $u_{\varepsilon}$ admits the asymptotic expansion

$$
\begin{aligned}
u_{\varepsilon}(x)=u_{0}(x)+ & a_{A}(\varepsilon) \mathfrak{w}_{A}(x)+a_{B}(\varepsilon) \mathfrak{w}_{B}(x) \\
& +U_{1,1}^{A}\left(\frac{x-A}{\varepsilon}\right) \varepsilon+V_{1,1}^{A}\left(\frac{x-A}{\varepsilon}\right) a_{A}(\varepsilon) \varepsilon+W_{1,1}^{A B}\left(\frac{x-A}{\varepsilon}\right) a_{B}(\varepsilon) \varepsilon \\
+ & U_{1,1}^{B}\left(\frac{x-B}{\varepsilon}\right) \varepsilon+V_{1,1}^{B}\left(\frac{x-B}{\varepsilon}\right) a_{B}(\varepsilon) \varepsilon+W_{1,1}^{B A}\left(\frac{x-B}{\varepsilon}\right) a_{A}(\varepsilon) \varepsilon+\mathcal{O}_{\mathrm{H}^{1}\left(\Omega_{\varepsilon}\right)}\left(\varepsilon^{2}\right) .
\end{aligned}
$$

The algorithm can be continued if one wishes to obtain a higher order expansion. The next step would be to define new correctors in slow variable in order to obtain the following term of the asymptotics:

$$
\begin{aligned}
u_{\varepsilon}=u_{0}+a_{A}(\varepsilon) \mathfrak{w}_{A}(x)+a_{B}(\varepsilon) \mathfrak{w}_{B}(x) \\
+\quad \sum_{k \geq 1}\left(U_{k, 1}^{A}\left(\frac{x-A}{\varepsilon}\right) \varepsilon^{k}+V_{k, 1}^{A}\left(\frac{x-A}{\varepsilon}\right) a_{A}(\varepsilon) \varepsilon^{k}+W_{k, 1}^{A B}\left(\frac{x-A}{\varepsilon}\right) a_{B}(\varepsilon) \varepsilon^{k}\right) \\
+\sum_{k \geq 1}\left(U_{k, 1}^{B}\left(\frac{x-B}{\varepsilon}\right) \varepsilon^{k}+V_{k, 1}^{B}\left(\frac{x-B}{\varepsilon}\right) a_{B}(\varepsilon) \varepsilon^{k}+W_{k, 1}^{B A}\left(\frac{x-B}{\varepsilon}\right) a_{A}(\varepsilon) \varepsilon^{k}\right) \\
\quad+\sum_{k \geq 2}\left(u_{k-1,1}^{A}(x) \varepsilon^{k}+v_{k-1,1}^{A}(x) a_{A}(\varepsilon) \varepsilon^{k}+w_{k-1,1}^{A B}(x) a_{B}(\varepsilon) \varepsilon^{k}\right) \\
\quad+\sum_{k \geq 2}\left(u_{k-1,1}^{B}(x) \varepsilon^{k}+v_{k-1,1}^{B}(x) a_{B}(\varepsilon) \varepsilon^{k}+w_{k-1,1}^{B A}(x) a_{A}(\varepsilon) \varepsilon^{k}\right)+\tilde{r}_{\varepsilon}^{3},
\end{aligned}
$$


and so on up to the desired precision and then apply the a priori estimates. The study made by V. Maz'ya and S. Poborchi for the case of a single inclusion in [17, Section 4.1.3] can directly be extended to two separated holes around two fixed points and the estimates (2.14) and (2.15) remains valid.

Another interest of this result comes from the theory of topology optimization. This theory initiated in $[19,8]$ receives a tremendously growing interest. In a recent paper [3], S. Amstuzt and M. Ciligot-Travain provide a unifying treatment of constrained shape and topology optimization problems. The variations of shapes using the so-called speed method as well as 'digging holes', the basic concept of sensitivity-based topology optimization, are viewed as a general way to perturb domains. To derive the optimality condition, Amstuzt and Ciligot-Travain make a crucial structure assumption (formulae (8) and (9) in their work [3]): an arbitrary number of topological variations should be allowed at the same time, in other words one should be able to justify the leading terms of an asymptotic expansion of a cost function for an arbitrary number of holes dug simultaneously in the domain. However, the large literature on topological optimization deals with a single perturbation. It is then important to check their assumption: for Neumann boundary conditions, it is a consequence of the works of Maz'ya and Mochdan [13, 14]. Here, we can validate their assumption in the more difficult case of Dirichlet boundary conditions in dimension two. It is a corollary of Theorem 3.1: it suffices to write the leading terms in the expansion (3.9). This term is given by (3.6) and it turns out that it is exactly the superposition of the leading terms of the contribution of each holes considered independently. The interaction appears at higher order in the series in $1 / \ln \varepsilon$ that defined the fractions $a_{A}$ and $a_{B}$ in $\ln \varepsilon$. The passage to an arbitrary number of inclusions is straightforward: the main difference is that the adapted scale $h_{\varepsilon}$ has to be computed by solving a larger linear system than (3.4).

\subsection{Numerical illustration}

We use the same function $f$ as in the case of one inclusion. We choose

$$
A=(-0.5,0.5) \quad \text { and } \quad B=(0.5,0.5) \text {. }
$$

The solution $u_{\varepsilon}$ of problem 4.1 with $\varepsilon=1 / 1.5^{7} \simeq 0.0585$ is computed by a finite element method and given in Figure 8(a). The first remainder $r_{\varepsilon}^{0}=u_{\varepsilon}-u_{0}$ is given in Figure 8(b).

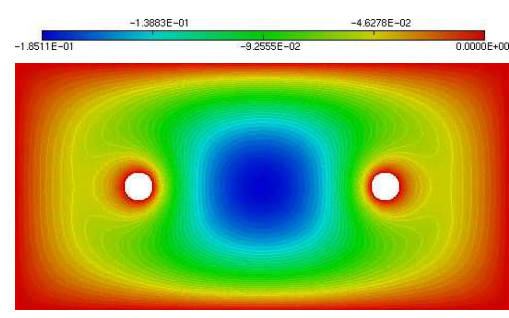

(a) $u_{\varepsilon}$

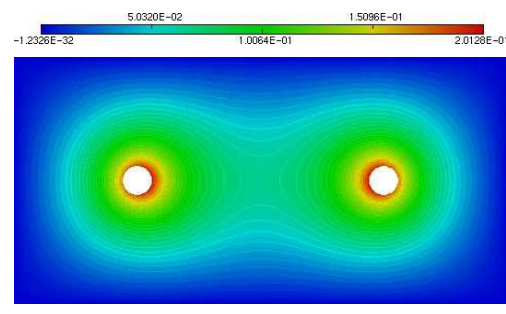

(b) $r_{\varepsilon}^{0}=u_{\varepsilon}-u_{0}$

Figure 8: First term approximation

The soution $w_{A}$ of problem (3.3) is given in Figure 9(a). The second term of the expansion $a_{A}(\varepsilon) \mathfrak{w}_{A}+a_{B}(\varepsilon) \mathfrak{w}_{B}$ is computed in Figure $9(\mathrm{c})$. The effect of the two terms approximation is illustrated in Figure 10. Table 2 gives the error norms when we aprroximate $u_{\varepsilon}$ by the first terms of its expansion. 


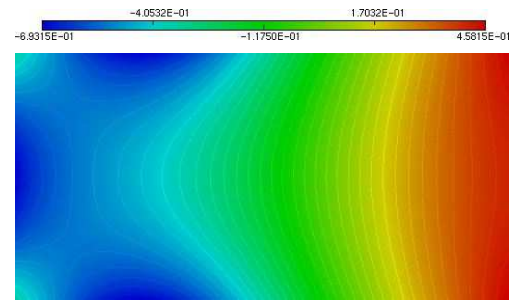

(a) $w_{A}$

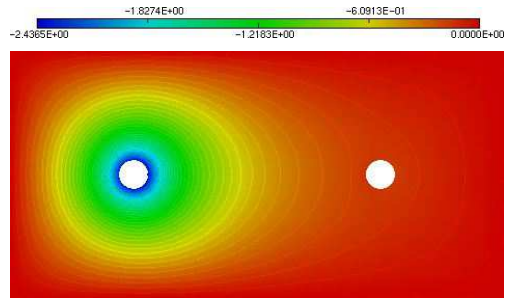

(b) $\mathfrak{w}_{A}$

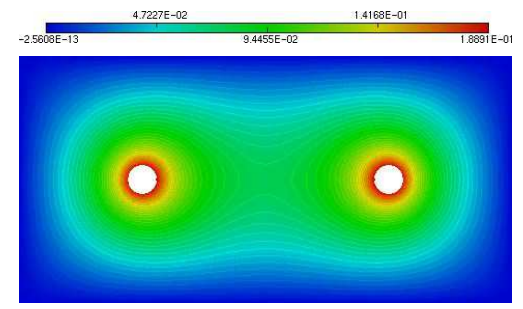

(c) $a_{A}(\varepsilon) \mathfrak{w}_{A}+a_{B}(\varepsilon) \mathfrak{w}_{B}$

Figure 9: Second term of the expansion

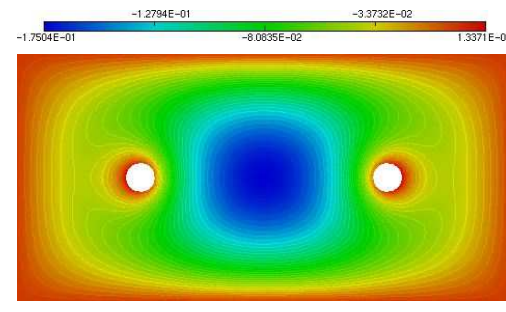

(a) $u_{0}+a_{A}(\varepsilon) \mathfrak{w}_{A}+a_{B}(\varepsilon) \mathfrak{w}_{B}$

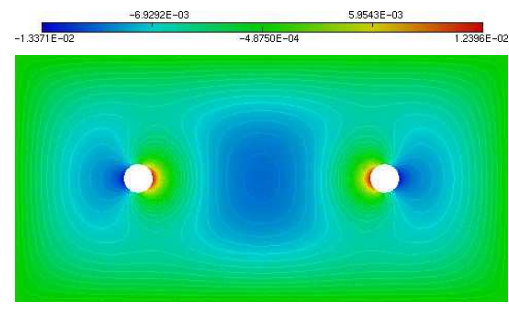

(b) $u_{\varepsilon}-u_{0}-a_{A}(\varepsilon) \mathfrak{w}_{A}-a_{B}(\varepsilon) \mathfrak{w}_{B}$

Figure 10: Second order approximation

\begin{tabular}{|c|c|c|}
\cline { 2 - 3 } \multicolumn{1}{c|}{} & $u_{\varepsilon}-u_{0}$ & $u_{\varepsilon}-u_{0}-a_{A}(\varepsilon) \mathfrak{w}_{A}-a_{B}(\varepsilon) \mathfrak{w}_{B}$ \\
\hline $\mathrm{L}^{2}$-norm & $8.323 \mathrm{e}-2$ & $7.76 \mathrm{e}-3$ \\
\hline $\mathrm{H}^{1}$-norm & $4.2756 \mathrm{e}-1$ & $4.946 \mathrm{e}-2$ \\
\hline
\end{tabular}

Table 2: Error norms

\section{Two moderately close inclusions case}

We consider the Dirichlet boundary value problem

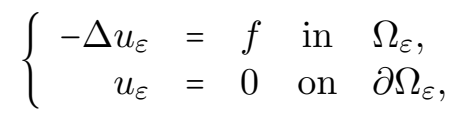

set in the domain:

$$
\Omega_{\varepsilon}=\Omega_{0} \sqrt{\omega_{\varepsilon}^{-} \cup \omega_{\varepsilon}^{+}}, \quad \text { with } \quad \omega_{\varepsilon}^{ \pm}=\mathcal{B}\left(x_{\varepsilon}^{ \pm}, \varepsilon\right) \quad \text { and } \quad x_{\varepsilon}^{ \pm}= \pm \varepsilon^{\alpha} \mathbf{d},
$$

as illustrated in Figure 11. We assume that $\mathbf{d}$ is a unit vector. 


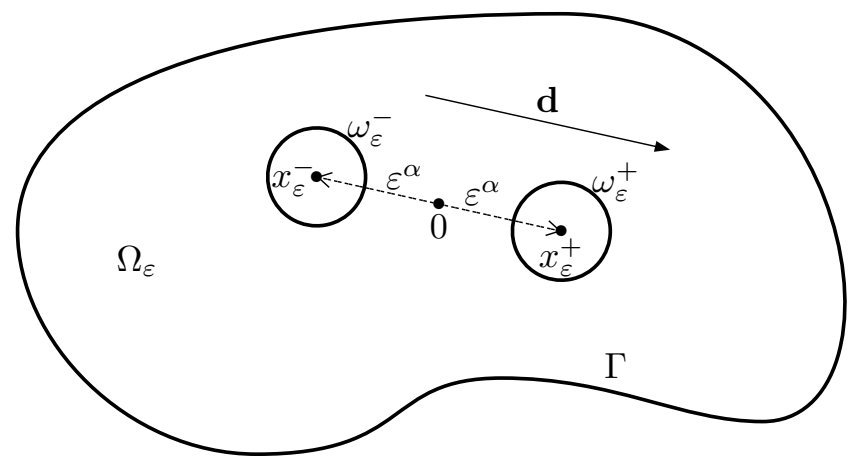

Figure 11: The perturbed domain

\subsection{Useful relations}

In the construction of the asymptotics expansion, we need to estimate terms where are involved distances between the center of the inclusion $x_{\varepsilon}^{ \pm}$and a point $x$ on the exterior boundary $\partial \Omega_{0}$ or on the boundary of the other inclusion $\omega_{\varepsilon}^{\mp}$. This subsection put together these estimates.

Let $x \in \partial \Omega_{0}$, then we can write

$$
\begin{aligned}
\left|x-x_{\varepsilon}^{ \pm}\right|^{2} & =|x|^{2}\left(1 \mp 2 \varepsilon^{\alpha} \frac{\langle x, \mathbf{d}\rangle}{|x|^{2}}+\frac{\varepsilon^{2 \alpha}}{|x|^{2}}\right) \\
\ln \left|x-x_{\varepsilon}^{ \pm}\right| & =\ln |x|+\sum_{k \geq 1} \tilde{B}_{k}^{ \pm}(x) \varepsilon^{\alpha k}, \quad \text { with } \quad \tilde{B}_{1}^{ \pm}=\mp \frac{\langle x, \mathbf{d}\rangle}{|x|^{2}} .
\end{aligned}
$$

Let $x \in \partial \omega_{\varepsilon}^{ \pm}$, there exists $X \in \mathcal{B}(0,1)$ such that $x= \pm\left(\varepsilon^{\alpha} \mathbf{d}+\varepsilon X\right)$ and we have

$$
\begin{aligned}
\left|x-x_{\varepsilon}^{\mp}\right|^{2} & =4 \varepsilon^{2 \alpha}\left(1+\varepsilon^{1-\alpha}\langle\mathbf{d}, X\rangle+\frac{\varepsilon^{2-2 \alpha}}{4}\right), \\
\ln \left|x-x_{\varepsilon}^{\mp}\right| & =\ln \left(2 \varepsilon^{\alpha}\right)+\sum_{k \geq 1} \tilde{a}_{k}(X) \varepsilon^{(1-\alpha) k} .
\end{aligned}
$$

Let us make the functions $\tilde{a}_{k}$ more explicit. We denote $h=\varepsilon^{1-\alpha} \cos \theta+\frac{1}{4} \varepsilon^{2-2 \alpha}$, with $\theta$ be such that $\cos \theta=\langle\mathbf{d}, X\rangle$, then

$$
\begin{aligned}
\ln (1+h) & =\sum_{k \geq 1} \frac{(-1)^{k+1}}{k} h^{k}=\sum_{k \geq 1} \frac{(-1)^{k+1}}{k} \sum_{j=0}^{k} C_{k}^{k-j} \cos ^{k-j} \theta \frac{\varepsilon^{(1-\alpha)(j+k)}}{4^{j}} \\
& =\sum_{m \geq 1} \varepsilon^{(1-\alpha) m} \sum_{\left[\frac{m}{2}\right] \leq k \leq m}(-1)^{k+1} \frac{(k-1) !}{(m-k) !(2 k-m) !} \frac{\cos ^{2 k-m} \theta}{4^{m-k}},
\end{aligned}
$$

with $m=j+k$. Using the change of variables $p=m-k$, we have:

$$
\sum_{k=\left[\frac{m}{2}\right]}^{m}(-1)^{k+1} \frac{(k-1) !}{(2 k-m) !(m-k) !} \frac{\cos ^{2 k-m} \theta}{2^{2 m-2 k}}=\sum_{p=0}^{\left[\frac{m}{2}\right]}(-1)^{m-p+1} \frac{(m-p-1) !}{j !(m-2 j) !} \frac{\cos ^{m-2 p} \theta}{2^{2 p}} .
$$

Using the Tchebychev polynomial functions (see [1, Chap. 22]), we have

$$
\frac{m}{2} \sum_{p=0}^{\left[\frac{m}{2}\right]}(-1)^{p} \frac{(m-p-1) !}{j !(m-2 p) !} 2^{m-2 p} \cos ^{m-2 p} \theta=\cos (m \theta)
$$


Consequently,

$$
\ln (1+h)=\sum_{m \geq 1} \varepsilon^{(1-\alpha) m} \frac{(-1)^{m+1}}{m} \frac{\cos (m \theta)}{2^{m-1}},
$$

and the function $a_{k}$ defined in (4.4) reads

$$
\tilde{a}_{k}(X)=\frac{(-1)^{k+1}}{k} \frac{\cos (k \theta)}{2^{k-1}} .
$$

We observe that

$$
\int_{\partial \mathcal{B}(0,1)} \tilde{a}_{k}=0
$$

\subsection{First term in slow variable}

As in the cases of one inclusion or two separated inclusions, we look for coefficients $a_{ \pm}(\varepsilon)$ and $b(\varepsilon)$ such that the remainder $r_{\varepsilon}^{1}$ defined by

$$
u_{\varepsilon}(x)=u_{0}(x)+a_{+}(\varepsilon) \ln \left|x-x_{\varepsilon}^{+}\right|+a_{-}(\varepsilon) \ln \left|x-x_{\varepsilon}^{-}\right|+b(\varepsilon) w(x)+r_{\varepsilon}^{1}(x),
$$

with $w$ defined in (2.5), is reduced with respect to $r_{\varepsilon}^{0}=u_{\varepsilon}-u_{0}$. The remainder $r_{\varepsilon}^{1}$ satisfies

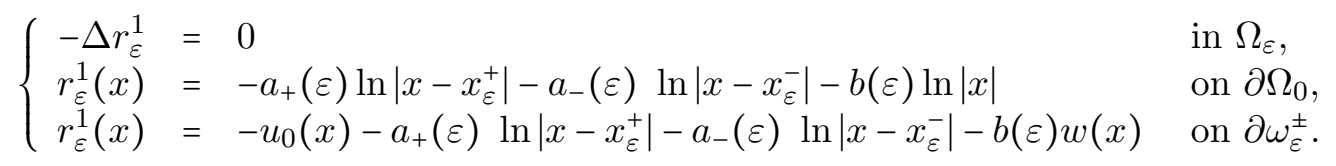

For $x \in \partial \Omega_{0}$, we have

$$
r_{\varepsilon}^{1}(x)=\mathcal{O}(1) \quad \Leftrightarrow \quad a_{+}(\varepsilon)+a_{-}(\varepsilon)+b(\varepsilon)=\mathcal{O}(1) .
$$

For $x=\varepsilon^{\alpha} \mathbf{d}+\varepsilon X \in \partial \omega_{\varepsilon}^{+}$, with $X \in \mathcal{B}(0,1)$, we have

$$
r_{\varepsilon}^{1}(x)=-u_{0}(0)+\mathcal{O}\left(\varepsilon^{\alpha}\right)-a_{+}(\varepsilon) \ln |\varepsilon X|-a_{-}(\varepsilon) \ln \left|2 \varepsilon^{\alpha} \mathbf{d}+\varepsilon X\right|-b(\varepsilon)\left(w(0)+\mathcal{O}\left(\varepsilon^{\alpha}\right)\right) .
$$

Consequently

$$
r_{\varepsilon}^{1}(x)=\mathcal{O}(1) \quad \Leftrightarrow \quad u_{0}(0)+a_{+}(\varepsilon) \ln \varepsilon+a_{-}(\varepsilon) \ln 2 \varepsilon^{\alpha}+w(0) b(\varepsilon)=\mathcal{O}(1) .
$$

We obtain the similar condition on $\partial \omega_{\varepsilon}^{-}$:

$$
r_{\varepsilon}^{1}(x)=\mathcal{O}(1) \quad \Leftrightarrow \quad u_{0}(0)+a_{+}(\varepsilon) \ln 2 \varepsilon^{\alpha}+a_{-}(\varepsilon) \ln \varepsilon+w(0) b(\varepsilon)=\mathcal{O}(1) .
$$

Hence we solve the linear system in the unknowns $\left(a_{ \pm}(\varepsilon), b(\varepsilon)\right)$ :

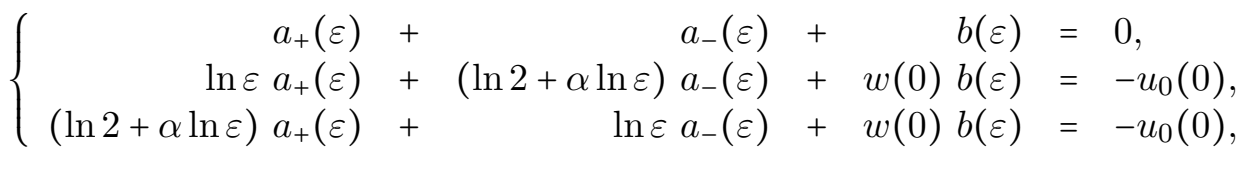

to set

$$
a_{+}(\varepsilon)=a_{-}(\varepsilon)=\frac{u_{0}(0)}{2 w(0)-\ln 2-(1+\alpha) \ln \varepsilon} \quad \text { and } \quad b(\varepsilon)=\frac{-2 u_{0}(0)}{2 w(0)-\ln 2-(1+\alpha) \ln \varepsilon} .
$$

As in (2.6) for the case of one inclusion, a new scale appears:

$$
h_{\varepsilon}=\frac{1}{2 w(0)-\ln 2-(1+\alpha) \ln \varepsilon}=\frac{-1}{(1+\alpha) \ln \varepsilon}+\mathcal{O}\left(\frac{1}{\ln ^{2} \varepsilon}\right) \quad \text { as } \quad \varepsilon \rightarrow 0 .
$$


A notable point is that the coefficient $\alpha$ measuring the distance between the two inclusion appears as a multiplicative factor in the leading term of the asymptotic. The situations of sections 3 and 4 are drastically different.

We introduce the normalized corrector $\mathfrak{w}_{\varepsilon}$ as

$$
\mathfrak{w}_{\varepsilon}(x)=\ln \left|x-x_{\varepsilon}^{+}\right|+\ln \left|x-x_{\varepsilon}^{-}\right|-2 w(x),
$$

with $w$ defined by (2.5). Then we approximate $u_{\varepsilon}$ by

$$
u_{\varepsilon}(x)=u_{0}(x)+u_{0}(0) \mathfrak{w}_{\varepsilon}(x) h_{\varepsilon}+\tilde{r}_{\varepsilon}^{1}(x) .
$$

\subsection{Second terms}

We check that the remainder $\tilde{r}_{\varepsilon}^{1}$ satisfies

$$
\left\{\begin{aligned}
-\Delta \tilde{r}_{\varepsilon}^{1} & =0 & & \text { in } \Omega_{\varepsilon} \\
\tilde{r}_{\varepsilon}^{1} & =-u_{0}(0) \mathfrak{w}_{\varepsilon} h_{\varepsilon} & & \text { on } \partial \Omega_{0} \\
\tilde{r}_{\varepsilon}^{1} & =-u_{0}-u_{0}(0) \mathfrak{w}_{\varepsilon} h_{\varepsilon} & & \text { on } \partial \omega_{\varepsilon}^{ \pm}
\end{aligned}\right.
$$

In order to estimate the first reminder $\tilde{r}_{\varepsilon}^{1}$, we examine its traces on $\Omega_{\varepsilon}$, that is on $\partial \Omega_{0}$ and on $\partial \omega_{\varepsilon}^{ \pm}$. For this, we use relations established in Subsection 4.1.

For any $x \in \partial \Omega_{0}$, we can write

$$
\tilde{r}_{\varepsilon}^{1}(x)=\sum_{k \geq 2}\left(B_{k}^{+}(x)+B_{k}^{-}(x)\right) h_{\varepsilon} \varepsilon^{\alpha k}, \quad \forall x \in \partial \Omega_{0},
$$

with $B_{k}^{ \pm}=-u_{0}(0) \tilde{B}_{k}^{ \pm}$and $\tilde{B}_{k}^{ \pm}$defined in (4.3). We have

$$
\left\|\tilde{r}_{\varepsilon}^{1}\right\|_{\mathrm{H}^{1 / 2}\left(\partial \Omega_{0}\right)}=\mathcal{O}\left(\frac{\varepsilon^{2 \alpha}}{\ln \varepsilon}\right) .
$$

Let us now look at the trace on the inclusions $\omega_{\varepsilon}^{ \pm}$. Let $x \in \partial \omega_{\varepsilon}^{ \pm}$, and $X \in \mathcal{B}(0,1)$ be such that $x= \pm\left(\varepsilon^{\alpha} \mathbf{d}+\varepsilon X\right)$. We have

$$
\ln \left|x-x_{\varepsilon}^{ \pm}\right|=\ln \varepsilon
$$

Then

$$
\tilde{r}_{\varepsilon}^{1}(x)=-u_{0}(x)-u_{0}(0)\left(\ln \varepsilon+\ln \left|x-x_{\varepsilon}^{\mp}\right|-2 w(x)\right) h_{\varepsilon} .
$$

Using Subsection 4.1, we can write relation (4.4)

$$
\ln \left|x-x_{\varepsilon}^{\mp}\right|=\ln \left(2 \varepsilon^{\alpha}\right)+\sum_{k \geq 1} \tilde{a}_{k}(X) \varepsilon^{(1-\alpha) k},
$$

with $\tilde{a}_{k}$ defined in (4.5) and satisfying (4.6). By interior elliptic regularity, $u_{0}$ and $w$ are analytic in the vicinity of 0 . Then

$$
\begin{aligned}
u_{0}(x) & =u_{0}(0)+\sum_{n \geq 1} D^{n} u_{0}(0)[x, \ldots, x] \\
& =u_{0}(0)+\sum_{j+k \geq 1}( \pm 1)^{j+k} C_{j+k}^{j} D^{j+k} u_{0}(0)[\underbrace{X, \ldots, X}_{j \text { times }}, \underbrace{\mathbf{d}, \ldots, \mathbf{d}}_{k \text { times }}] \varepsilon^{j+\alpha k}, \\
w(x) & =w(0)+\sum_{n \geq 1} D^{n} w(0)[x, \ldots, x],
\end{aligned}
$$


in the vicinity of 0 . For the convenience, we denote

$$
\begin{aligned}
a_{k}(X) & =-u_{0}(0) \tilde{a}_{k}(X), \\
u_{j, k}^{ \pm}(X) & =( \pm 1)^{j+k+1} C_{j+k}^{j} D^{j+k} u_{0}(0)\left[X^{j}, \mathbf{d}^{k}\right], \\
w_{j, k}^{ \pm}(X) & =( \pm 1)^{j+k} 2 u_{0}(0) C_{j+k}^{j} D^{j+k} w(0)\left[X^{j}, \mathbf{d}^{k}\right],
\end{aligned}
$$

with $a_{k}$ defined in (4.5). Then the remainder $\tilde{r}_{\varepsilon}^{1}$ reads

$$
\tilde{r}_{\varepsilon}^{1}(x)=\sum_{j+k>0} u_{j, k}^{ \pm}(X) \varepsilon^{j+\alpha k}+\sum_{k \geq 1} a_{k}(X) h_{\varepsilon} \varepsilon^{(1-\alpha) k}+\sum_{j+k>0} w_{j, k}^{ \pm}(X) h_{\varepsilon} \varepsilon^{j+\alpha k}, \quad \forall x \in \partial \omega_{\varepsilon}^{ \pm} .
$$

As for the case of one inclusion, the mean value property insures that

$$
\int_{\partial \mathcal{B}(0,1)} u_{j, k}^{ \pm}=0 \quad \text { and } \quad \int_{\partial \mathcal{B}(0,1)} w_{j, k}^{ \pm}=0 .
$$

Now we need to lift each boundary condition appearing in (4.9) and (4.10).

- The functions $B_{k}^{ \pm}$introduced in (4.3) to estimate the remainder on the exterior boundary as given in (4.9) generate correctors $b_{k}^{ \pm}$defined by

$$
\left\{\begin{aligned}
-\Delta b_{k}^{ \pm} & = & & \text {in } \Omega_{0} \\
b_{k}^{ \pm} & =B_{k}^{ \pm} & & \text {on } \partial \Omega_{0} .
\end{aligned}\right.
$$

These correctors do not satisfy the Dirichlet condition on the boundary of the inclusion $\partial \omega_{\varepsilon}^{ \pm}$and then generate errors on these boundaries.

- We now proceed as in the case of one inclusion when we lift $u_{k}$ and $w_{k}$. We notice that the mean value property is satisfied since

$$
\int_{\partial \mathcal{B}(0,1)} a_{k}=0, \quad \int_{\partial \mathcal{B}(0,1)} u_{j, k}^{ \pm}=0 \quad \text { and } \int_{\partial \mathcal{B}(0,1)} w_{j, k}^{ \pm}=0 .
$$

Then Lemma 2.1 (point 3) can be applied. We set $A_{k}, U_{j, k}^{ \pm}$and $V_{j, k}^{ \pm}$the solution of problem (2.4) with $v=a_{k}, v=u_{j, k}^{ \pm}$and $v=w_{j, k}^{ \pm}$respectively:

$$
\left\{\begin{array} { c l } 
{ - \Delta A _ { k } = 0 } & { \text { in } \mathbb { R } ^ { 2 } \backslash \omega , } \\
{ A _ { k } = a _ { k } } & { \text { on } \partial \omega , }
\end{array} \quad \left\{\begin{array} { c l } 
{ - \Delta U _ { j , k } ^ { \pm } = 0 } & { \text { in } \mathbb { R } ^ { 2 } \backslash \omega , } \\
{ U _ { j , k } ^ { \pm } = u _ { j , k } ^ { \pm } } & { \text { on } \partial \omega , }
\end{array} \quad \left\{\begin{array}{cl}
-\Delta W_{j, k}^{ \pm}=0 & \text { in } \mathbb{R}^{2} \backslash \omega, \\
W_{j, k}^{ \pm}=w_{j, k}^{ \pm} & \text {on } \partial \omega .
\end{array}\right.\right.\right.
$$

These functions have similar decomposition as in (2.8) and (2.9).

We then approximate $u_{\varepsilon}$ by

$$
\begin{aligned}
& u_{\varepsilon}(x)=u_{0}(x)+u_{0}(0) \mathfrak{w}_{\varepsilon}(x) h_{\varepsilon}+\sum_{k \geq 2}\left(b_{k}^{+}(x)+b_{k}^{-}(x)\right) h_{\varepsilon} \varepsilon^{\alpha k} \\
& +\sum_{k \geq 1}\left(A_{k}\left(\frac{x-x_{\varepsilon}^{+}}{\varepsilon}\right)+A_{k}\left(\frac{x-x_{\varepsilon}^{-}}{\varepsilon}\right)\right) h_{\varepsilon} \varepsilon^{(1-\alpha) k}+\sum_{j+k>0}\left(U_{j, k}^{+}\left(\frac{x-x_{\varepsilon}^{+}}{\varepsilon}\right)+U_{j, k}^{-}\left(\frac{x-x_{\varepsilon}^{-}}{\varepsilon}\right)\right) \varepsilon^{j+\alpha k} \\
& +\sum_{j+k>0}\left(W_{j, k}^{+}\left(\frac{x-x_{\varepsilon}^{+}}{\varepsilon}\right)+W_{j, k}^{-}\left(\frac{x-x_{\varepsilon}^{-}}{\varepsilon}\right)\right) h_{\varepsilon} \varepsilon^{j+\alpha k}+\tilde{r}_{\varepsilon}^{2}(x) .
\end{aligned}
$$




\subsection{Third terms}

Let us study now the remainder $\tilde{r}_{\varepsilon}^{2}$. It satisfies

$$
\left\{\begin{array}{rlrl}
-\Delta \tilde{r}_{\varepsilon}^{2}= & & \text { in } \Omega_{\varepsilon}, \\
\tilde{r}_{\varepsilon}^{2}(x)= & -\sum_{k \geq 1}\left(A_{k}\left(\frac{x-x_{\varepsilon}^{+}}{\varepsilon}\right)+A_{k}\left(\frac{x-x_{\varepsilon}^{-}}{\varepsilon}\right)\right) h_{\varepsilon} \varepsilon^{(1-\alpha) k} & \\
& -\sum_{j+k>0}\left(U_{j, k}^{+}\left(\frac{x-x_{\varepsilon}^{+}}{\varepsilon}\right)+U_{j, k}^{-}\left(\frac{x-x_{\varepsilon}^{-}}{\varepsilon}\right)\right) \varepsilon^{j+\alpha k} & \\
& -\sum_{j+k>0}\left(W_{j, k}^{+}\left(\frac{x-x_{\varepsilon}^{+}}{\varepsilon}\right)+W_{j, k}^{-}\left(\frac{x-x_{\varepsilon}^{-}}{\varepsilon}\right)\right) h_{\varepsilon} \varepsilon^{j+\alpha k} & & \text { for } x \in \partial \Omega_{0}, \\
\tilde{r}_{\varepsilon}^{2}= & -\sum_{k \geq 2}\left(b_{k}^{+}+b_{k}^{-}\right) h_{\varepsilon} \varepsilon^{\alpha k} & \text { on } \partial \omega_{\varepsilon}^{ \pm} .
\end{array}\right.
$$

- Let us study the trace on $\partial \Omega_{0}$. According to their decomposition like in (2.8) and (2.9), the traces $T A_{k}^{ \pm}, T U_{j, k}$ and $T W_{j, k}$ on $\partial \Omega_{0}$ of the functions $A_{k}\left(\frac{x-x_{\varepsilon}^{ \pm}}{\varepsilon}\right), U_{j, k}^{ \pm}\left(\frac{x-x_{\varepsilon}^{ \pm}}{\varepsilon}\right)$ and $W_{j, k}^{ \pm}\left(\frac{x-x_{\varepsilon}^{ \pm}}{\varepsilon}\right)$ satisfy

$\left\|T A_{k}^{ \pm}\right\|_{\mathrm{H}^{1 / 2}\left(\partial \Omega_{0}\right)}=\mathcal{O}(\varepsilon), \quad\left\|T U_{j, k}^{ \pm}\right\|_{\mathrm{H}^{1 / 2}\left(\partial \Omega_{0}\right)}=\mathcal{O}(\varepsilon) \quad$ and $\quad\left\|T W_{j, k}^{ \pm}\right\|_{\mathrm{H}^{1 / 2}\left(\partial \Omega_{0}\right)}=\mathcal{O}(\varepsilon)$.

We now lift these functions in slow variables and define $\hat{a}_{k}^{ \pm}, \hat{u}_{j, k}^{ \pm}$and $\hat{w}_{j, k}^{ \pm}$as the solution of

$$
\left\{\begin{aligned}
-\Delta \hat{v} & =0 & & \text { in } \Omega_{0}, \\
\hat{v} & =-\frac{1}{\varepsilon} T V & & \text { on } \partial \Omega_{0},
\end{aligned}\right.
$$

with $T V=T A_{k}\left(\frac{x-x_{\varepsilon}^{ \pm}}{\varepsilon}\right), T U_{j, k}^{ \pm}\left(\frac{x-x_{\varepsilon}^{ \pm}}{\varepsilon}\right)$ and $T V_{j, k}^{ \pm}\left(\frac{x-x_{\varepsilon}^{ \pm}}{\varepsilon}\right)$ respectively.

- To lift the boundary condition on $\partial \omega_{\varepsilon}^{ \pm}$, we proceed as in the first step of the construction of the asymptotic expansion (see also the study of the remainder $\tilde{r}_{\varepsilon}^{3}$ in the case of one inclusion). The function $b_{k}^{ \pm}$have a value at 0 which is a priori not 0 . We can lift the boundary condition by considering the function

$$
-\sum_{k \geq 2}\left(b_{k}^{+}(0)+b_{k}^{-}(0)\right) \mathfrak{w}_{\varepsilon}(x) h_{\varepsilon}^{2} \varepsilon^{\alpha k} .
$$

We can read $u_{\varepsilon}$ :

$$
\begin{aligned}
& u_{\varepsilon}(x)=u_{0}(x)+u_{0}(0) \mathfrak{w}_{\varepsilon}(x) h_{\varepsilon} \\
& \quad+\sum_{k \geq 2}\left(b_{k}^{+}(x)+b_{k}^{-}(x)\right) h_{\varepsilon} \varepsilon^{\alpha k}-\sum_{k \geq 2}\left(b_{k}^{+}(0)+b_{k}^{-}(0)\right) \mathfrak{w}_{\varepsilon}(x) h_{\varepsilon}^{2} \varepsilon^{\alpha k} \\
& +\sum_{k \geq 1}\left(A_{k}\left(\frac{x-x_{\varepsilon}^{+}}{\varepsilon}\right)+A_{k}\left(\frac{x-x_{\varepsilon}^{-}}{\varepsilon}\right)\right) h_{\varepsilon} \varepsilon^{(1-\alpha) k}+\sum_{k \geq 2}\left(\hat{a}_{k-1}^{+}(x)+\hat{a}_{k-1}^{-}(x)\right) h_{\varepsilon} \varepsilon^{k-\alpha(k-1)} \\
& +\sum_{j+k>0}\left(U_{j, k}^{+}\left(\frac{x-x_{\varepsilon}^{+}}{\varepsilon}\right)+U_{j, k}^{-}\left(\frac{x-x_{\varepsilon}^{-}}{\varepsilon}\right)\right) \varepsilon^{j+\alpha k}+\sum_{j+k>1, j \geq 1}\left(\hat{u}_{j-1, k}^{+}(x)+\hat{u}_{j-1, k}^{-}(x)\right) \varepsilon^{j+\alpha k} \\
& +\sum_{j+k>0}\left(W_{j, k}^{+}\left(\frac{x-x_{\varepsilon}^{+}}{\varepsilon}\right)+W_{j, k}^{-}\left(\frac{x-x_{\varepsilon}^{-}}{\varepsilon}\right)\right) h_{\varepsilon} \varepsilon^{j+\alpha k}+\sum_{j+k>1, j \geq 1}\left(\hat{w}_{j-1, k}^{+}(x)+\hat{w}_{j-1, k}^{-}(x)\right) h_{\varepsilon} \varepsilon^{j+\alpha k} \\
& +\tilde{r}_{\varepsilon}^{3}(x) .
\end{aligned}
$$




\subsection{Antsatz}

We inject the remainder $\tilde{r}_{\varepsilon}^{3}$ in the equation and obtain

$$
\left\{\begin{array}{rlrl}
-\Delta \tilde{r}_{\varepsilon}^{3}= & & \text { in } \Omega_{\varepsilon}, \\
\tilde{r}_{\varepsilon}^{3}= & \sum_{k \geq 2}\left(b_{k}^{+}(0)+b_{k}^{-}(0)\right) \mathfrak{w}_{\varepsilon} h_{\varepsilon}^{2} \varepsilon^{\alpha k} & \text { on } \partial \Omega_{0}, \\
\tilde{r}_{\varepsilon}^{3}= & -\sum_{k \geq 2}\left(\hat{a}_{k-1}^{+}(x)+\hat{a}_{k-1}^{-}(x)\right) h_{\varepsilon} \varepsilon^{k-\alpha(k-1)} & \\
& -\sum_{j+k>1, j \geq 2}\left(\hat{u}_{j-1, k}^{+}+\hat{u}_{j-1, k}^{-}\right) \varepsilon^{j+\alpha k}-\sum_{j+k>1, j \geq 2}\left(\hat{w}_{j-1, k}^{+}+\hat{w}_{j-1, k}^{-}\right) h_{\varepsilon} \varepsilon^{j+\alpha k} & & \text { on } \partial \omega_{\varepsilon}^{ \pm} .
\end{array}\right.
$$

We have now to reiterate the previous step with the new functions.

This justify the building of the antsatz

$$
\begin{aligned}
u_{\varepsilon}(x) & =u_{0}(x)+u_{0}(0) \mathfrak{w}_{\varepsilon}(x) h_{\varepsilon}+\sum_{i \geq 0, j \geq 1, k \geq 0} c_{i, j, k} \mathfrak{w}_{\varepsilon}(x) h_{\varepsilon}^{i} \varepsilon^{j+\alpha k} \\
& +\sum_{i \geq 1, k \geq 2} \beta_{i, k}\left(b_{k}^{+}(x)+b_{k}^{-}(x)\right) h_{\varepsilon}^{i} \varepsilon^{\alpha k}+\sum_{i \geq 1, k \geq 2} \beta_{i, k}\left(b_{k}^{+}(0)+b_{k}^{-}(0)\right) \mathfrak{w}_{\varepsilon}(x) h_{\varepsilon}^{i+1} \varepsilon^{\alpha k} \\
+ & \sum_{i \geq 0, j \geq 0, k \geq 1}\left(A_{i, j, k}\left(\frac{x-x_{\varepsilon}^{+}}{\varepsilon}\right)+A_{i, j, k}\left(\frac{x-x_{\varepsilon}^{-}}{\varepsilon}\right)\right) h_{\varepsilon}^{i} \varepsilon^{j+k(1-\alpha)}+\sum_{i \geq 0, j \geq 0, k \geq 1} \hat{a}_{i, j, k}(x) h_{\varepsilon}^{i} \varepsilon^{j+1+k(1-\alpha)} \\
& +\sum_{i \geq 0, j+k>0}\left(V_{i, j, k}^{+}\left(\frac{x-x_{\varepsilon}^{+}}{\varepsilon}\right)+V_{i, j, k}^{-}\left(\frac{x-x_{\varepsilon}^{-}}{\varepsilon}\right)\right) h_{\varepsilon}^{i} \varepsilon^{j+\alpha k}+\sum_{i \geq 0, j+k>0}\left(\hat{v}_{i, j, k}^{+}(x)+\hat{v}_{i, j, k}^{-}(x)\right) h_{\varepsilon}^{i} \varepsilon^{j+1+\alpha k}
\end{aligned}
$$

To justify it, we proceed exactly as in Section 2.6.

\subsection{Numerical illustration}

We use the same test as in the case of one inclusion. The solution $u_{\varepsilon}$ of problem 4.1 with $\varepsilon=1 / 1.5^{7} \simeq 0.0585$ and $\alpha=0.8$ is computed by a finite element method and given in Figure 12(a). With these parameters, we have

$$
h_{\varepsilon} \simeq-0.067 \quad \text { and } \quad \frac{-1}{(1+\alpha) \ln \varepsilon} \simeq 0.049 .
$$

The first remainder $r_{\varepsilon}^{0}=u_{\varepsilon}-u_{0}$ is given in Figure 12(b). The second term of the expansion

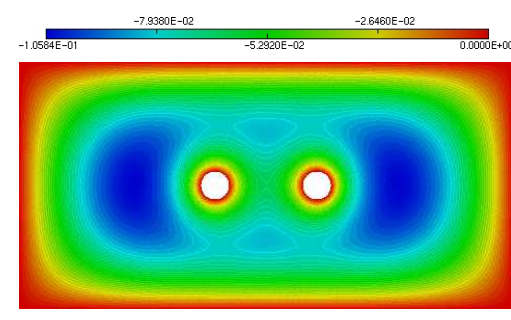

(a) $u_{\varepsilon}$

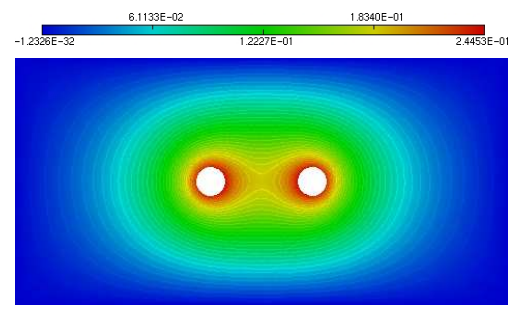

(b) $r_{\varepsilon}^{0}=u_{\varepsilon}-u_{0}$

Figure 12: First term approximation

$u_{0}(0) \mathfrak{w}_{\varepsilon} h_{\varepsilon}$ is computed in Figure 13. The effect of the two terms approximation is illustrated in Figures 14 and 15. 


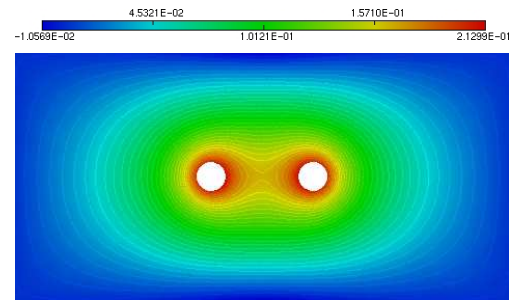

(a) $u_{0}(0) \mathfrak{w} h_{\varepsilon}$

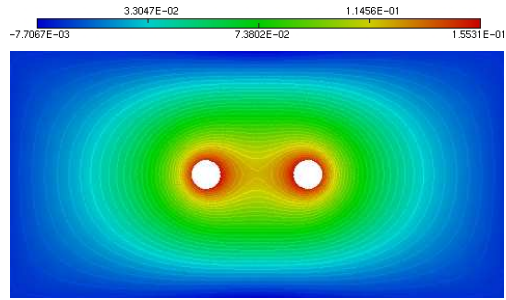

(b) $-u_{0}(0) \mathfrak{w} /((1+\alpha) \ln \varepsilon)$

Figure 13: Second term of the expansion

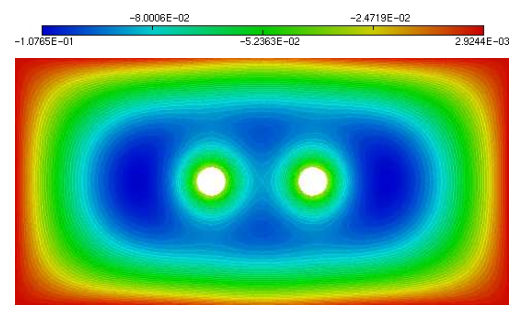

(a) $u_{0}+u_{0}(0) \mathfrak{w} h_{\varepsilon}$

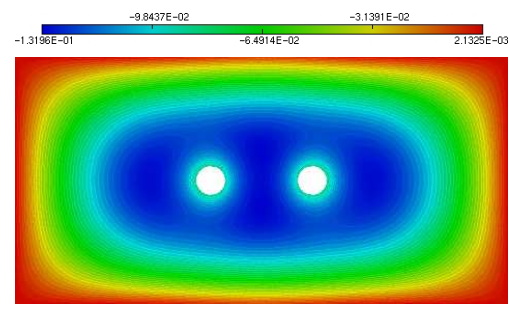

(b) $u_{0}-u_{0}(0) \mathfrak{w} /((1+\alpha) \ln \varepsilon)$

Figure 14: Two term expansion

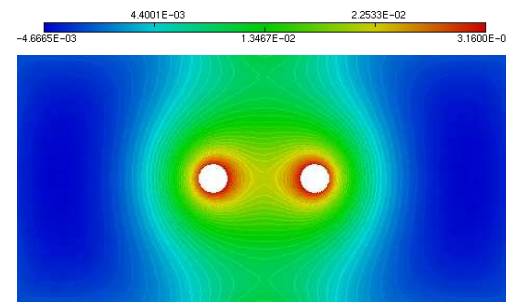

(a) $u_{\varepsilon}-u_{0}-u_{0}(0) \mathfrak{w} h_{\varepsilon}$

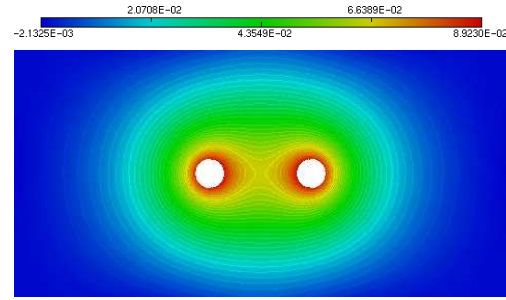

(b) $u_{\varepsilon}-u_{0}+u_{0}(0) \mathfrak{w} / \ln \varepsilon$

Figure 15: Second order approximation

\begin{tabular}{|c|c|c|c|}
\cline { 2 - 4 } \multicolumn{1}{c|}{} & $u_{\varepsilon}-u_{0}$ & $u_{\varepsilon}-u_{0}-u_{0}(0) \mathfrak{w} h_{\varepsilon}$ & $u_{\varepsilon}-u_{0}+u_{0}(0) \mathfrak{w} /((1+\alpha) \ln \varepsilon)$ \\
\hline $\mathrm{L}^{2}$-norm & $1.0858 \mathrm{e}-1$ & $1.235 \mathrm{e}-2$ & $3.753 \mathrm{e}-2$ \\
\hline $\mathrm{H}^{1}$-norm & $4.9162 \mathrm{e}-1$ & $6.715 \mathrm{e}-2$ & $1.7603 \mathrm{e}-1$ \\
\hline
\end{tabular}

Table 3: Error norms

\section{References}

[1] M. Abramowitz, I. A. Stegun. Handbook of mathematical functions with formulas, graphs, and mathematical tables, volume 55 of National Bureau of Standards Applied Mathematics Series. For sale by the Superintendent of Documents, U.S. Government Printing Office, Washington, D.C. 1964.

[2] C. Amrouche, V. Girault, J. Giroire. Dirichlet and Neumann exterior problems for the $n$-dimensional Laplace operator: an approach in weighted Sobolev spaces. $J$. Math. Pures Appl. (9) 76(1) (1997) 55-81. 
[3] S. Amstutz, M. Ciligot-Travain. Optimality conditions for shape and topology optimization subject to a cone constraint. SIAM J. Control Optim. 48(6) (2010) 4056-4077.

[4] V. Bonnaillie-Nö̈l, M. Dambrine, S. Tordeux, G. Vial. Interactions between moderately close inclusions for the Laplace equation. Math. Models Methods Appl. Sci. 19(10) (2009) 1853-1882.

[5] M. Dambrine, G. Vial. Influence of a boundary perforation on the Dirichlet energy. Control Cybernet. 34(1) (2005) 117-136.

[6] M. Dambrine, G. Vial. A multiscale correction method for local singular perturbations of the boundary. M2AN Math. Model. Numer. Anal. 41(1) (2007) 111-127.

[7] E. Gagliardo. Caratterizzazioni delle tracce sulla frontiera relative ad alcune classi di funzioni in $n$ variabili. Rend. Sem. Mat. Univ. Padova 27 (1957) 284-305.

[8] S. Garreau, P. Guillaume, M. Masmoudi. The topological asymptotic for PDE systems: the elasticity case. SIAM J. Control Optim. 39(6) (2001) 1756-1778 (electronic).

[9] J. Giroire. Étude de quelques problèmes aux limites extérieurs et résolution par équations intégrales. Thèse de doctorat d'état, Université Pierre et Marie Curie, Paris VI 1987.

[10] A. M. IL'IN. Matching of asymptotic expansions of solutions of boundary value problems, volume 102 of Translations of Mathematical Monographs. American Mathematical Society, Providence, RI 1992.

[11] T. Lewiński, J. SokoŁowski. Topological derivative for nucleation of non-circular voids. The Neumann problem. In Differential geometric methods in the control of partial differential equations (Boulder, CO, 1999), volume 268 of Contemp. Math., pages 341-361. Amer. Math. Soc., Providence, RI 2000.

[12] D. Martin. MÉLina, bibliothèque de calculs éléments finis. http://perso.univ-rennes1.fr/daniel.martin/melina (2007).

[13] V. Maz'Ya, A. Movchan. Uniform asymptotic formulae for Green's functions in singularly perturbed domains. J. Comput. Appl. Math. 208(1) (2007) 194-206.

[14] V. Maz'ya, A. Movchan. Asymptotic treatment of perforated domains without homogenization. Math. Nachr. 283(1) (2010) 104-125.

[15] V. Maz'ya, S. Nazarov, B. Plamenevskij. Asymptotic theory of elliptic boundary value problems in singularly perturbed domains. Vol. I, volume 111 of Operator Theory: Advances and Applications. Birkhäuser Verlag, Basel 2000.

[16] V. Maz'ya, S. Nazarov, B. Plamenevskij. Asymptotic theory of elliptic boundary value problems in singularly perturbed domains. Vol. II, volume 112 of Operator Theory: Advances and Applications. Birkhäuser Verlag, Basel 2000.

[17] V. G. Maz'YA, S. V. Poborchi. Differentiable functions on bad domains. World Scientific Publishing Co. Inc., River Edge, NJ 1997. 
[18] S. A. Nazarov, J. SokoŁowski. Asymptotic analysis of shape functionals. J. Math. Pures Appl. (9) 82(2) (2003) 125-196.

[19] J. SokoŁowski, A. Żochowski. On the topological derivative in shape optimization. SIAM J. Control Optim. 37(4) (1999) 1251-1272 (electronic). 